\title{
Multivariant model of martensitic microstructure in thin films
}

\author{
Y.C. Shu*, J.H. Yen \\ Institute of Applied Mechanics, National Taiwan University, No. 1, Sector 4, Roosevelt Road, Taipei 106, Taiwan, ROC
}

Received 22 October 2007; received in revised form 10 April 2008; accepted 10 April 2008

Available online 26 May 2008

\begin{abstract}
A novel multivariant model of martensite is developed through competing energetics to describe the coarsening, refinement and selection of the microstructure. In contrast with the conventional phase-field methods, a new set of field variables motivated by the hierarchical structure of multirank laminates is employed to represent each variant. As a result, the energy-well structure can be expressed explicitly in an elegant and unified fashion. The framework is applied to the investigation of pattern formation in martensitic thin films with trigonal symmetry. Various intriguing patterns are predicted and are found to be in good agreement with those observed in experiments. In addition, film orientations and patterns necessary to achieve large actuation strains are suggested for dome-shaped and tunnelshaped microactuators. It is found that the resulting morphologies evolve with coherent interfaces under various loading conditions. This suggests that compatible walls provide a low-energy path during evolution, and the understanding of them leads to novel strategies of large strain actuation.
\end{abstract}

(C) 2008 Acta Materialia Inc. Published by Elsevier Ltd. All rights reserved.

Keywords: Martensite; Pattern formation; Thin films

\section{Introduction}

A martensitic material undergoes a first-order, diffusionless, solid-to-solid phase transformation during which there is a sudden change in the crystal structure at a certain temperature. Crystals going through a thermoelastic martensitic transformation often exhibit the shape-memory effect - a phenomenon where deformation suffered below a critical temperature can be recovered on heating. This property enables these alloys to be used for a variety of applications and makes them attractive candidates for smart materials, since they function as actuators as well as sensors [1-3]. Recently, several proposed designs for creating tiny machines have suggested that the characteristic microstructure patterns can be exploited as device elements [4]. This requires the design of devices that can take full advantage of the inherent microstructure to achieve this

\footnotetext{
* Corresponding author. Tel.: +88623366 5627; fax: +88623366 5628. E-mail address: yichung@spring.iam.ntu.edu.tw (Y.C. Shu).
}

goal, which in turn raises many fundamental issues in martensitic materials.

Much work has addressed the formation and description of various aspects of microstructure in bulk crystals but, for a number of reasons, little attention was paid to thin films until recently. A typical film has a characteristic geometry where one dimension is much smaller than the other two with large surfaces. Consequently, a dimensional constraint appears as a new length scale comparable to that of microstructure. Moreover, martensitic crystals are highly anisotropic and nonlinear due to phase transformation. All of these facts have made it difficult to develop suitable theories to describe their behavior in slender structures. Bhattacharya and James [5] employed ideas similar to the notion of $\Gamma$-convergence to derive a theory of martensitic single crystal films, and Shu [6] extended it to polycrystalline films. They showed that the microstructure in thin films can be different from that in the bulk, and this enables a novel strategy that directly uses aspects of this microstructure for building new microactuators $[7,8]$. Bringing these ideas to practical applications requires a thorough understanding and characterization of the 
microstructure and its evolution under stress. This calls for an appropriate model that not only can capture the spirit of the Bhattacharya-James theory but also can serve as a useful tool for evaluating various conditions in the design process. The goal of this paper is to develop such a model and validate it. A strategy exploiting the microstructure and orientation of the film for performance optimization of microactuators is also recommended here.

The key feature of a martensitic phase transformation is the microstructure it generates. The high-temperature austenite phase is cubic, while the low-temperature martensite phase has less symmetry. This gives rise to symmetryrelated variants that are identical crystal lattices of martensite with different orientations. The crystal is able to take a specific shape by making a fine-scaled mixture of different variants to accommodate the deformation. However, these variants cannot be arbitrarily mixed; instead, each interface separating different variants has a well-defined crystallographic orientation such that rows of atoms are unbroken across it. As a result, variants of martensite form highly intricate and very characteristic patterns at a length scale much smaller than the size of the sample. To explain these observed patterns, the crystallographic theory of martensite was proposed by Wechsler et al. [9], as well as by Bowles and MacKenzie [10], in the early 1950s. However, this theory is purely kinematic in origin and involves a priori the twinning mode, leading to difficulty in extending it to other circumstances. Ball and James [11] developed a theoretical approach to predict the fine microstructure based on the relaxation of a nonlinear energy function. Khachaturyan [12] and Roitburd [13], on the other hand, used a different theory based on linear elasticity and the Fourier technique for predicting the morphology of crystal microstructures. Both theories are based on energy minimization and can recover the results of the early crystallographic theory. The link between these two was established by Kohn [14], who showed that the latter is the geometrically linear analogue of the former. Bhattacharya and Kohn [15] and Shu and Bhattacharya [16] extended the Ball-James theory to the case of polycrystals.

As analytic solutions obtained from the above-mentioned theories are available only for some simplified problems, this article adopts another perspective by proposing a multivariant framework suitable for simulating patterns of microstructure under a variety of boundary conditions. This problem has been studied by Khachaturyan, Roytburd, Salje, Saxena, Lookman and their collaborators for martensites [17-24], and by Chen and his co-workers for ferroelectrics $[25,26]$. Other important contributions for microstructure simulation include the works [27-31]. All of them use the conventional time-dependent GinzburgLandau (TDGL) model, and choose a suitable set of order parameters and the special polynomial expansions of these parameters at high orders for a particular transformation. Instead, a new set of field variables is introduced to represent each variant here [32]. This approach is motivated by the hierarchical structure of multirank laminates con- structed by Bhattacharya [33] for establishing the rule of mixtures. It provides the advantage of expressing the energy-well structure of martensitic variants in a unified fashion, irrespective of the different types of transformation under consideration. Besides, only two parameters are needed in the proposed framework. One is related to the energetic cost due to formation of the interface and the other is the cost due to the deviation from the ground state energy.The extensions of the present framework for simulating domain patterns in ferroelectrics and ferromagnetic shape-memory alloys are currently underway [34,35]. Moreover, this idea of employing laminated domain patterns has been applied to the investigation of ferroelectric switching by Shu et al. [36] and Yen et al. [37].

This article is organized as follows: It starts with the use of energetics to describe the coarsening, refinement and selection of microstructure of martensite in Section 2. This phenomenon has been observed in other physical systems, including self-organized nanoscale patterns in a binary epitaxial monolayer [38] and nanomesa and nanowell formation in a Langmuir-Blodgett ferroelectric polymeric film [39]. The framework is then applied to the investigation of pattern formation in martensitic thin films in Section 3 . Ti-Ni in the R-phase state is chosen as the model material. The R-phase transformation is martensitic and thermoelastic, and is characterized by a rhombohedral distortion of the parent B2 structure [40]. While it yields a relatively small shape change, its temperature hysteresis vs. strain is an order of magnitude smaller than that of the monoclinic phase. Thus, it is suitable in transducer applications. Indeed, microactuators exploiting the Rphase transformation of $\mathrm{Ti}-\mathrm{Ni}$ films have been developed recently with operation frequency around $100 \mathrm{~Hz}$ [41]. Various self-accommodation patterns are predicted from simulations. They are satisfied with the interface conditions of the Bhattacharya-James thin film theory [5], and some of them are found to be in good agreement with those observed in experiments. In the last part of Section 3, the model is applied to the design of large strain microactuators by targeting the optimal microstructures and film orientations. Conclusions are drawn in Section 4.

\section{Framework and formulation}

\subsection{Transformation strain}

Consider a single crystal of austenite and choose this as the reference configuration. The crystal occupies the region $\Omega \subset \mathbb{R}^{3}$ in its reference configuration. The displacement and linear strain of the crystal are described by the functions $\mathbf{u}$ and $\boldsymbol{\varepsilon}$. Both are related by

$\boldsymbol{\varepsilon}[\mathbf{u}]=\frac{1}{2}\left\{\nabla \mathbf{u}+(\nabla \mathbf{u})^{\mathrm{T}}\right\}$

The austenite has stress-free strain $\boldsymbol{\varepsilon}^{(0)}=\mathbf{0}$. As it is cooled, it transforms to martensite. In shape-memory alloys, the austenite lattice has cubic symmetry, while the martensite 
lattice has lesser symmetry, such as tetragonal, trigonal, orthorhombic or monoclinic symmetry. This gives rise to $N$ symmetry-related variants of martensite. The transformation from the austenite to the $i$ th variant of martensite is described by the transformation (Bain) strain $\boldsymbol{\varepsilon}^{(i)}$. It can be determined from the change of symmetry and lattice parameters. For example, in the case of cubic-to-trigonal transformation, $N=4$, and the transformation strain of each variant is described by

$$
\begin{aligned}
& \boldsymbol{\varepsilon}^{(1)}=(\alpha-\delta) \mathbf{I}+3 \delta \mathbf{p}^{(1)} \otimes \mathbf{p}^{(1)} \\
& \boldsymbol{\varepsilon}^{(2)}=(\alpha-\delta) \mathbf{I}+3 \delta \mathbf{p}^{(2)} \otimes \mathbf{p}^{(2)} \\
& \boldsymbol{\varepsilon}^{(3)}=(\alpha-\delta) \mathbf{I}+3 \delta \mathbf{p}^{(3)} \otimes \mathbf{p}^{(3)} \\
& \boldsymbol{\varepsilon}^{(4)}=(\alpha-\delta) \mathbf{I}+3 \delta \mathbf{p}^{(4)} \otimes \mathbf{p}^{(4)}
\end{aligned}
$$

where $\mathbf{I}$ is the identity tensor, $\alpha$ and $\delta$ are material constants, and each $\mathbf{p}^{(i)}$ is a unit vector directing along one of the body diagonals of a cube, i.e.

$$
\begin{array}{ll}
\mathbf{p}^{(1)}=\frac{1}{\sqrt{3}}\left(\mathbf{e}_{1}+\mathbf{e}_{2}+\mathbf{e}_{3}\right), & \mathbf{p}^{(2)}=\frac{1}{\sqrt{3}}\left(-\mathbf{e}_{1}+\mathbf{e}_{2}+\mathbf{e}_{3}\right) \\
\mathbf{p}^{(3)}=\frac{1}{\sqrt{3}}\left(\mathbf{e}_{1}-\mathbf{e}_{2}+\mathbf{e}_{3}\right), & \mathbf{p}^{(4)}=\frac{1}{\sqrt{3}}\left(-\mathbf{e}_{1}-\mathbf{e}_{2}+\mathbf{e}_{3}\right)
\end{array}
$$

and $\left\{\mathbf{e}_{1}, \mathbf{e}_{2}, \mathbf{e}_{3}\right\}$ is an orthonormal basis parallel to the cubic lattice of the austenite. Above in Eq. (2) the notation $\mathbf{a} \otimes \mathbf{b}$ denotes the tensor product of any two vectors $\mathbf{a}$ and $\mathbf{b}$, and its matrix representation is $(\mathbf{a} \otimes \mathbf{b})_{i j}=a_{i} b_{j}$ for $i, j=1,2,3$, where $a_{i}$ and $b_{j}$ are components of $\mathbf{a}$ and $\mathbf{b}$ in the basis $\left\{\mathbf{e}_{1}, \mathbf{e}_{2}, \mathbf{e}_{3}\right\}$. To explain Eq. (2), notice that the transformation strain $\varepsilon^{(1)}$ can also be rewritten as

$$
\begin{aligned}
\boldsymbol{\varepsilon}^{(1)} & =\mathbf{U}^{(1)}-\mathbf{I} \\
& =\left\{\eta_{2} \mathbf{p}^{(1)} \otimes \mathbf{p}^{(1)}+\eta_{1}\left(\mathbf{q}^{(1)} \otimes \mathbf{q}^{(1)}+\mathbf{r}^{(1)} \otimes \mathbf{r}^{(1)}\right)\right\}-\mathbf{I}
\end{aligned}
$$

where $\eta_{1}=\alpha-\delta+1, \eta_{2}=\alpha+2 \delta+1$, and the vectors $\mathbf{q}^{(1)}$ and $\mathbf{r}^{(1)}$ are any pairs such that $\left\{\mathbf{p}^{(1)}, \mathbf{q}^{(1)}, \mathbf{r}^{(1)}\right\}$ forms an orthonormal set of vectors in $\mathbb{R}^{3}$. Thus, $\mathbf{U}^{(1)}$ represents a distortion obtained by stretching the cubic lattice along the body diagonal direction $\mathbf{p}^{(1)}$. Similarly, other variants are obtained by stretching the same lattice along the rest of three body diagonal directions $\mathbf{p}^{(2)}, \mathbf{p}^{(3)}$ or $\mathbf{p}^{(4)}$, as demonstrated in Fig. 1. The expression of Bain strains for other cases such as tetragonal, orthorhombic and monoclinic martensitic crystals can be found in [16].

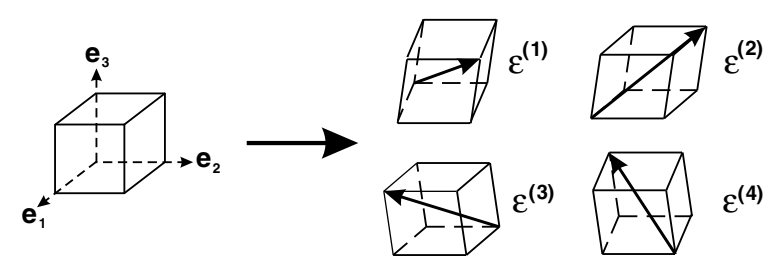

Fig. 1. Schematic of four trigonal variants described in terms of transformation strains $\varepsilon^{(1)}, \varepsilon^{(2)}, \varepsilon^{(3)}$ and $\varepsilon^{(4)}$. The crystal and reference bases are assumed to coincide together, and each arrow represents the stretching along one of the body diagonal directions of a cube.
Let $\Omega_{i} \subset \Omega$ be the region occupied by the $i$ th martensitic variant for $i=1,2, \ldots, N$. Thus, $\Omega_{i} \cap \Omega_{j}=\emptyset$ if $i \neq j$. Let $\varepsilon^{*}(\mathbf{x})$ be the locally inhomogeneous transformation strain field such that $\boldsymbol{\varepsilon}^{*}(\mathbf{x})=\boldsymbol{\varepsilon}^{(i)}$ if $\mathbf{x} \in \Omega_{i}$; or, equivalently,

$\boldsymbol{\varepsilon}^{*}(\mathbf{x})=\sum_{i=1}^{N} \gamma_{i}(\mathbf{x}) \boldsymbol{\varepsilon}^{(i)}$

where $\gamma_{i}(\mathbf{x})=1$ if $\mathbf{x} \in \Omega_{i}$ and $\gamma_{i}(\mathbf{x})=0$ otherwise. Alternatively, the transformation strain field $\boldsymbol{\varepsilon}^{*}(\mathbf{x})$ can be described by employing a set of field variables $\left\{\mu_{1}, \mu_{2}, \ldots, \mu_{N-1}\right\}$ whose elements $\mu_{i}$ are equal to either 0 or 1 . Indeed, set

$$
\begin{aligned}
& \gamma_{1}=\mu_{1} \\
& \gamma_{2}=\mu_{2}\left(1-\mu_{1}\right) \\
& \gamma_{3}=\mu_{3}\left(1-\mu_{1}\right)\left(1-\mu_{2}\right) \\
& \cdots \\
& \gamma_{N-1}=\mu_{N-1}\left(1-\mu_{1}\right) \cdots\left(1-\mu_{N-2}\right) \\
& \gamma_{N}=\left(1-\mu_{1}\right) \cdots\left(1-\mu_{N-2}\right)\left(1-\mu_{N-1}\right)
\end{aligned}
$$

in Eq. (4). Notice that when $\mu_{i}=1$ and $\mu_{1}=\mu_{2}=$ $\cdots=\mu_{i-1}=0, \gamma_{i}=1$ and $\gamma_{j}=0$ for $j \neq i$. If, however, all $\mu_{1}=\mu_{2}=\cdots=\mu_{N-1}=0$, then $\gamma_{N}=1$ and $\gamma_{j}=0$ for $j \neq N$. Thus, assigning discrete values to each of the field variables $\mu_{i}$ guarantees that at each point $\mathbf{x} \in \Omega$, only one of $\gamma_{i}$ is equal to 1 and the rest of them vanishes.

Introducing $\mu_{i}$ in Eq. (5) as the field variables, instead of the conventional choice $\gamma_{i}$, to express the transformation strain field $\varepsilon^{*}$ is motivated by the rule of mixtures proposed by Bhattacharya [33]. Simply speaking, suppose two variants $i$ and $j$ are twin-related, i.e. the corresponding transformation strains satisfy

$\boldsymbol{\varepsilon}^{(i)}-\boldsymbol{\varepsilon}^{(j)}=\frac{1}{2}(\mathbf{a} \otimes \mathbf{n}+\mathbf{n} \otimes \mathbf{a})$

for some vectors $\mathbf{a}$ and $\mathbf{n}$. The strain compatibility in Eq. (6) implies that a laminate microstructure can be constructed by alternating layers of these two variants with an overall strain

$\lambda \boldsymbol{\varepsilon}^{(i)}+(1-\lambda) \boldsymbol{\varepsilon}^{(j)}$

where $0 \leqslant \lambda \leqslant 1$ is the volume fraction of the $i$ th variant. If all the variants are twin-related satisfying Eq. (6), the rule of mixture indicates that a coherent rank $(N-1)$ laminate can be developed to accommodate any prescribed average $\left\langle\varepsilon^{*}\right\rangle$ with

$$
\begin{aligned}
\left\langle\boldsymbol{\varepsilon}^{*}\right\rangle= & \left\langle\gamma_{1}\right\rangle \boldsymbol{\varepsilon}^{(1)}+\left\langle\gamma_{2}\right\rangle \boldsymbol{\varepsilon}^{(2)}+\cdots+\left\langle\gamma_{N-1}\right\rangle \boldsymbol{\varepsilon}^{(N-1)}+\left\langle\gamma_{N}\right\rangle \boldsymbol{\varepsilon}^{(N)} \\
= & \lambda_{1} \boldsymbol{\varepsilon}^{(1)}+\lambda_{2}\left(1-\lambda_{1}\right) \boldsymbol{\varepsilon}^{(2)}+\cdots+\lambda_{N-1} \prod_{k=1}^{N-2}\left(1-\lambda_{k}\right) \boldsymbol{\varepsilon}^{(N-1)} \\
& +\prod_{k=1}^{N-1}\left(1-\lambda_{k}\right) \boldsymbol{\varepsilon}^{(N)}
\end{aligned}
$$

where the symbol $\langle\cdots\rangle$ denotes the average over the volume of a body, and $\lambda_{i}$ is the local volume fraction of the $i$ th-rank laminate, as illustrated in Fig. 2 (a rank-3 laminate in trigonal martensite). This result is important since laminated 


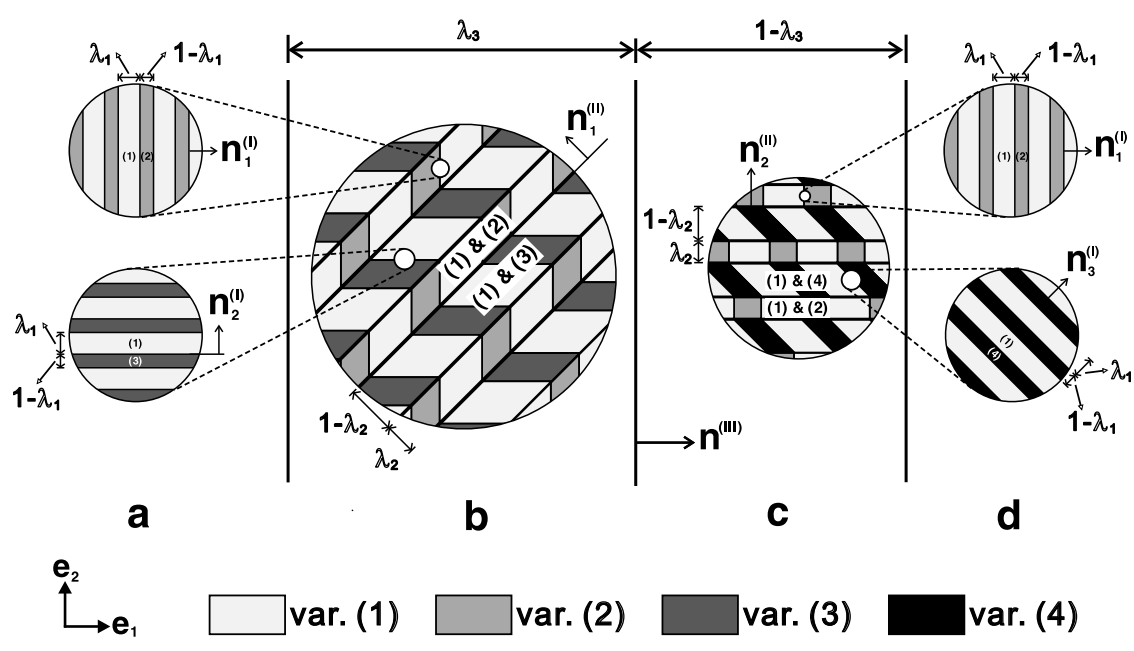

Fig. 2. Schematic representation of a compatible rank-3 laminate in trigonal martensite. Each variant is presented by a different gray level, as listed on the bottom. Note that the scales of different levels of laminates are assumed to be widely separated. The transition layers on the interfaces are not drawn here to avoid congestion.

microstructures are commonly observed in martensite [1]. It therefore raises a fundamental question about how the idea of multirank lamination is incorporated in a phasefield model of martensite. From the comparison between Eq. (5) and Eq. (8), it is suggested that the nonuniform field variables $\mu_{i}$ be used instead of $\gamma_{i}$ to describe the locally inhomogeneous transformation strain field $\varepsilon^{*}$. An advantage of using the proposed approach is that it provides a unified way for specifying the energy-well structure of martensite, and will be explained in Section 2.2.

Finally, the derivation of Eq. (8) is simply sketched in the case of trigonal martensite. First, it can be verified that all trigonal variants given by Eq. (2) are twin-related satisfying Eq. (6). A further investigation reveals that there are two types of twins classified by different interfacial normals: $\{100\}$ and $\{110\}$. Table 1 lists all possible compatible interfacial normals among different variants. Second, according to Eq. (7) and Table 1, variants (1) and (2) can form a rank-1 laminate by choosing $\mathbf{n}_{1}^{(\mathrm{I})}=(1,0,0)$, and variants (1) and (3) can form another rank-1 laminate with $\mathbf{n}_{2}^{(\mathrm{I})}=(0,1,0)$. Both are shown in Fig. 2a, where $\lambda_{1}$ is the volume fraction of variant (1). These two rank-1 laminates can further form a rank-2 laminate if they alternate in layers separated by parallel planes with unit normal $\mathbf{n}_{1}^{(\mathrm{II})}$, as demonstrated in Fig. $2 b$, where $\lambda_{2}$ is the volume fraction of layers comprising variants (1) and (2) in this case. This rank-2 laminate is legitimate if the scales of different levels of laminates are widely separated, and the interfacial normal $\mathbf{n}_{1}^{(\mathrm{II})}$ is oriented to satisfy Eq. (6) in the sense of average.

Table 1

Compatible interfacial normals in a bulk martensite with trigonal symmetry

\begin{tabular}{lllllll}
\hline Variants & 1,2 & 1,3 & 1,4 & 2,3 & 2,4 & 3,4 \\
\hline$\{100\}$ type & $(1,0,0)$ & $(0,1,0)$ & $(0,0,1)$ & $(0,0,1)$ & $(0,1,0)$ & $(1,0,0)$ \\
$\{110\}$ type & $(0,1,1)$ & $(1,0,1)$ & $(1,1,0)$ & $(-1,1,0)$ & $(-1,0,1)$ & $(0,-1,1)$ \\
\hline
\end{tabular}

In the present case, the average compatibility requires $\mathbf{n}_{1}^{(\mathrm{II})}=\left(\frac{-1}{\sqrt{2}}, \frac{1}{\sqrt{2}}, 0\right)$. Third, using an argument similar to the above, variants (1), (2) and (4) of trigonal martensite can also form another rank-2 laminate characterized by local volume fractions $\lambda_{1}$ and $\lambda_{2}$, as shown in Fig. $2 \mathrm{c}$ and $\mathrm{d}$. Now a rank-3 laminate can be constructed by alternating these two rank-2 laminates separated by an interface with unit normal $\mathbf{n}^{\text {(III) }}$, as shown in Fig. 2, where $\lambda_{3}$ is the volume fraction of layers containing variants (1), (2) and (3). Again, it is a coherent pattern if the average compatibility condition across the interface $\mathbf{n}^{(\mathrm{III})}$ can be satisfied, and we have $\mathbf{n}^{(\mathrm{III})}=(1,0,0)$. Employing Eq. (7) repeatedly over each level of laminates gives the overall strain of this rank-3 laminate as in Eq. (8) with $N=4$. Thus, by choosing $\lambda_{1}, \lambda_{2}$ and $\lambda_{3}$ suitably, any average of transformation strains can be achieved using coherent patterns of martensitic variants.

\subsection{Free energy}

The elastic energy density of martensite taking its heterogeneous microstructure into account is

$W^{\text {elas }}(\boldsymbol{\mu})=\frac{1}{2}\left[\boldsymbol{\varepsilon}-\boldsymbol{\varepsilon}^{*}(\boldsymbol{\mu})\right] \cdot \mathbf{C}\left[\boldsymbol{\varepsilon}-\boldsymbol{\varepsilon}^{*}(\boldsymbol{\mu})\right]$

where $\varepsilon^{*}(\boldsymbol{\mu})$ is given by Eq. (4) using Eq. (5), $\boldsymbol{\mu}=\left(\mu_{1}, \mu_{2}, \ldots, \mu_{N-1}\right)$ and $\mathbf{C}$ is the elastic modulus assumed to be the same for all phases. The elastic tensor $\mathbf{C}$ is positive-definite symmetric such that, for $i, j, k, l=1,2,3$

$C_{i j k l}=C_{i j l k}=C_{k l i j}$

where $C_{i j k l}$ is the component of $\mathbf{C}$ in some orthonormal basis. The compatible strain $\varepsilon$ is implicitly dependent on the field variable $\boldsymbol{\mu}$ and is determined by solving the mechanical equilibrium equation

$\nabla \cdot \boldsymbol{\sigma}=0, \quad \boldsymbol{\sigma}=\mathbf{C}\left[\boldsymbol{\varepsilon}-\boldsymbol{\varepsilon}^{*}(\boldsymbol{\mu})\right] \quad \forall \mathbf{x} \in \Omega$ 
subject to suitable boundary conditions. In Eq. (10) $\sigma$ is the symmetric elastic stress tensor.

As explained in Section 2.1, each $\mu_{i}$ has to take on either 0 or 1 such that $\boldsymbol{\varepsilon}^{*}(\boldsymbol{\mu})$ stands for one of the transformation strains. To enforce it, we introduce the notion of energy density $W^{\mathrm{ani}}(\boldsymbol{\mu})$, which is the energetic cost when the field variable $\boldsymbol{\mu}$ deviates from these two points. Specifically, one of the simplest forms of it to achieve this purpose is

$W^{\mathrm{ani}}(\boldsymbol{\mu})=\sum_{i=1}^{N-1} K_{i} \mu_{i}^{2}\left(1-\mu_{i}\right)^{2}$

where $K_{i}>0$ for $i=1, \ldots, N-1$. Thus, let $\boldsymbol{\mu}$ be relaxed to take on any value. Minimizing

$$
\begin{aligned}
W^{\text {ani }}(\boldsymbol{\mu})+W^{\text {elas }}(\boldsymbol{\mu})= & \sum_{i=1}^{N-1} K_{i} \mu_{i}^{2}\left(1-\mu_{i}\right)^{2}+\frac{1}{2}\left[\boldsymbol{\varepsilon}-\boldsymbol{\varepsilon}^{*}(\boldsymbol{\mu})\right] \\
& \cdot \mathbf{C}\left[\boldsymbol{\varepsilon}-\boldsymbol{\varepsilon}^{*}(\boldsymbol{\mu})\right]
\end{aligned}
$$

forces each $\mu_{i}$ to take on the value very close to either 0 or 1 if $K_{i}$ is sufficiently large. The zeros of ( $\left.W^{\text {ani }}+W^{\text {elas }}\right)$ define the tensorial directions along which the crystal is deformed most easily. Thus, this builds in the information that the crystal prefers certain spontaneous strain at a given temperature. Moreover, if there are no discrepancies among all martensitic variants, $K=K_{i}$ for each $i$, so that each martensitic variant owns an identical energy of the ground state.

Next, to describe the sharp interface separating different variants of martensite, we introduce the notion of interfacial energy density by

$W^{\text {int }}(\boldsymbol{\mu})=A|\nabla \boldsymbol{\mu}|^{2}$

where $A>0$ is called the exchange or gradient coefficient. From Eq. (12), $\boldsymbol{\mu}$ has been relaxed to continuously vary across the boundaries of martensitic variants. This energy density $W^{\text {int }}(\boldsymbol{\mu})$ penalizes changes in $\boldsymbol{\mu}$ and thus is interpreted as the energy of forming a martensitic interface. Such changes are supposed to be abrupt if $A$ is assumed to be very small. Further, according to Eq. (5), several different sets of $\mu_{i}$ may represent the same variant. However, our numerous numerical results in Section 3 have revealed that the adjacent variants typically select particular sets of $\mu_{i}$ to minimize the interfacial energy.

Under these hypotheses (sufficiently large $K$ and small $A$ ), we postulate that the pattern of martensitic variants at some fixed temperature below the critical temperature can be obtained by minimizing

$$
\mathscr{I}(\boldsymbol{\mu})=\int_{\Omega}\left\{W^{\text {int }}(\boldsymbol{\mu})+W^{\text {ani }}(\boldsymbol{\mu})+W^{\text {elas }}(\boldsymbol{\mu})-\boldsymbol{\sigma}^{0} \cdot \boldsymbol{\varepsilon}\right\} \mathrm{d} \mathbf{x}
$$

subject to the constraint Eq. (10). Above, $\sigma^{0}$ is the external stress induced owing to the applied traction on some parts of its boundary. It has to be divergence-free in the absence of body forces and consistent with the mechanical boundary conditions [42]. Note that if the boundary condition is specified by displacement on the whole part of the boundary, the last term $\boldsymbol{\sigma}^{0} \cdot \varepsilon$ in Eq. (13) is dropped.

The task here is to determine the field variable $\boldsymbol{\mu}$, which minimizes the free energy in Eq. (13) subject to the constraint Eq. (10). However, this is not an easy task due to the nonlinear optimization and the complexity in solving the elastic equilibrium equation for arbitrary finite shape of $\Omega$. In fact, Shu et al. [42] have proposed a modified boundary integral formalism to solve Eq. (10) to simulate the evolution of magnetic domains for magnetostrictive materials. However, the size of their simulation is restricted to the nanoscale. Fortunately, for a bulk martensite, the length scale of martensitic microstructure is typically much smaller than the body size. Thus, it is reasonable to assume the periodic boundary condition in Eq. (13) for simulating microstructure in bulk materials, i.e. $\boldsymbol{\mu}$ is periodic over a small cube $\Omega=\left(0, l_{0}\right)^{3}$, where $l_{0}$ is larger than the microstructure size but is smaller than the size of the body. Under this assumption, the elastic constraint Eq. (10) can be solved as follows. First, the elastic compatible strain $\varepsilon$ and transformation strain field $\varepsilon^{*}$ can be decomposed as the sum of macroscopically homogeneous state and microscopically nonuniform state

$\boldsymbol{\varepsilon}=\langle\boldsymbol{\varepsilon}\rangle+\boldsymbol{\varepsilon}^{\prime}, \quad \boldsymbol{\varepsilon}^{*}=\left\langle\boldsymbol{\varepsilon}^{*}\right\rangle+\boldsymbol{\varepsilon}^{* \prime}$

where $\varepsilon^{\prime}$ is the perturbed inhomogeneous strain and is related with the displacement $\mathbf{u}^{\prime}$ through Eq. (1). Both $\mathbf{u}^{\prime}$ and $\boldsymbol{\varepsilon}^{\prime}$ are periodic and can be obtained by solving

$\nabla \cdot \boldsymbol{\sigma}^{\prime}=0, \quad \boldsymbol{\sigma}^{\prime}=\mathbf{C}\left[\varepsilon^{\prime}\left[\mathbf{u}^{\prime}\right]-\varepsilon^{* \prime}(\boldsymbol{\mu})\right]$

at fixed $\boldsymbol{\mu}$. The solution of $\boldsymbol{\sigma}^{\prime}$ can be achieved explicitly using the technique of Fourier transform and its derivation is provided in Appendix A. The homogeneous state $\langle\boldsymbol{\sigma}\rangle=\mathbf{C}\left[\langle\varepsilon\rangle-\left\langle\varepsilon^{*}\right\rangle\right]$, however, depends on the specific types of boundary conditions. For instance, the complete stress field can be expressed as

$$
\begin{aligned}
& \boldsymbol{\sigma}=\mathbf{C}\left[\boldsymbol{\varepsilon}^{0}-\left\langle\boldsymbol{\varepsilon}^{*}\right\rangle\right]+\boldsymbol{\sigma}^{\prime}, \quad \text { if }\langle\boldsymbol{\varepsilon}\rangle=\boldsymbol{\varepsilon}^{0} \text { is imposed } \\
& \boldsymbol{\sigma}=\boldsymbol{\sigma}^{0}+\boldsymbol{\sigma}^{\prime}, \quad \text { if }\langle\boldsymbol{\sigma}\rangle=\boldsymbol{\sigma}^{0} \text { is imposed }
\end{aligned}
$$

\subsection{Evolution of microstructure under driving forces}

Applying the variation in $\boldsymbol{\mu}$ to Eq. (13) gives

$$
\begin{aligned}
\delta \mathscr{I}(\boldsymbol{\mu})= & -\int_{\Omega}\left(\mathbf{F}^{\mathrm{int}}+\mathbf{F}^{\mathrm{ani}}+\mathbf{F}^{\mathrm{elas}}\right) \cdot \delta \boldsymbol{\mu} \mathrm{d} \mathbf{x}+\int_{\partial \Omega} 2 A \frac{\partial \boldsymbol{\mu}}{\partial \mathbf{n}} \cdot \delta \boldsymbol{\mu} \mathrm{d} S \\
& +\int_{\Omega}\left(\boldsymbol{\sigma}-\boldsymbol{\sigma}^{0}\right) \cdot \delta \boldsymbol{\varepsilon}[\mathbf{u}] \mathrm{d} \mathbf{x}
\end{aligned}
$$

where $\partial \Omega$ is the boundary of $\Omega, \mathbf{n}$ is the outward unit vector to $\partial \Omega, \mathrm{d} S$ is the surface element and the driving forces

$$
\begin{aligned}
& \mathbf{F}^{\text {int }}=2 A \nabla^{2} \boldsymbol{\mu} \\
& \mathbf{F}^{\text {ani }}=-\frac{\partial W^{\text {ani }}(\boldsymbol{\mu})}{\partial \boldsymbol{\mu}} \\
& \mathbf{F}^{\text {elas }}=\mathbf{C}\left[\boldsymbol{\varepsilon}-\boldsymbol{\varepsilon}^{*}(\boldsymbol{\mu})\right] \cdot \frac{\partial \boldsymbol{\varepsilon}^{*}(\boldsymbol{\mu})}{\partial \boldsymbol{\mu}}
\end{aligned}
$$


The surface integral term in Eq. (18) originates from

$$
\begin{aligned}
\delta \int_{\Omega} A|\nabla \boldsymbol{\mu}|^{2} \mathrm{~d} \mathbf{x} & =\int_{\Omega} 2 A[\nabla \boldsymbol{\mu} \cdot \nabla(\delta \boldsymbol{\mu})] \mathrm{d} \mathbf{x} \\
& =\int_{\Omega} 2 A\left\{\nabla \cdot[\delta \boldsymbol{\mu} \cdot(\nabla \boldsymbol{\mu})]-\delta \boldsymbol{\mu} \cdot \nabla^{2} \boldsymbol{\mu}\right\} \mathrm{d} \mathbf{x} \\
& =-\int_{\Omega} \mathbf{F}^{\mathrm{int}} \cdot \delta \boldsymbol{\mu} \mathrm{d} \mathbf{x}+\int_{\partial \Omega} 2 A \frac{\partial \boldsymbol{\mu}}{\partial \mathbf{n}} \cdot \delta \boldsymbol{\mu} \mathrm{d} S
\end{aligned}
$$

The last integral term in Eq. (18) can be shown to be

$\int_{\Omega}\left(\boldsymbol{\sigma}-\boldsymbol{\sigma}^{0}\right) \cdot \delta \varepsilon[\mathbf{u}] \mathrm{d} \mathbf{x}=0$

and the derivation of Eq. (20) is provided in Appendix B.

Now the necessary condition for minimizing the total free energy $\mathscr{I}(\boldsymbol{\mu})$ is the first variation of it has to be equal to zero, i.e. $\delta \mathscr{I}(\boldsymbol{\mu})=0$. In other words, from Eq. (18)

$\mathbf{F}(\boldsymbol{\mu})=-\frac{\delta}{\delta \boldsymbol{\mu}} \mathscr{I}(\boldsymbol{\mu})=\mathbf{F}^{\mathrm{int}}+\mathbf{F}^{\text {ani }}+\mathbf{F}^{\text {elas }}=\mathbf{0}$

$\left\{\begin{array}{l}\frac{\partial \boldsymbol{\mu}}{\partial \mathbf{n}}(\mathbf{x})=\mathbf{0} \quad \forall \mathbf{x} \in \partial \Omega \text { (nonperiodic setting) } \\ \boldsymbol{\mu} \text { is periodic over a cube (periodic setting) }\end{array}\right.$

where $\mathbf{F}$ is the total thermodynamic driving force defined by the negative of the variational derivative of the free energy with respect to the field variable $\boldsymbol{\mu}$. Physically, $\mathbf{F}^{\text {int }}$ is the driving force for coarsening microstructure, $\mathbf{F}^{\text {ani }}$ is the driving force for selecting variants and $\mathbf{F}^{\text {elas }}$ is the driving force for refining microstructure to accommodate the specified boundary constraints.

The nonzero $\mathbf{F}$ defined by Eq. (21) provides a force driving the microstructure to evolve. The simplest form to describe the evolution of microstructure is given by

$\frac{\partial \boldsymbol{\mu}}{\partial t}=-M \frac{\delta \mathscr{I}}{\delta \boldsymbol{\mu}}=M \mathbf{F}$

where $M>0$ is the mobility. It is obvious that $\mathbf{F}=\mathbf{0}$ can be viewed as a particular case of Eq. (22), giving the steady- state solution. In fact, the evolution of $\boldsymbol{\mu}$ described by Eq. (22) implies the decrease of the total free energy in Eq. (13). This can be understood by considering

$$
\begin{aligned}
\frac{\mathrm{d} \mathscr{I}}{\mathrm{d} t} & =\int_{\Omega}\left\{\frac{\partial W^{\mathrm{int}}}{\partial \boldsymbol{\mu}}+\frac{\partial W^{\mathrm{ani}}}{\partial \boldsymbol{\mu}}+\frac{\partial W^{\text {elas }}}{\partial \boldsymbol{\mu}}-\boldsymbol{\sigma}^{0} \cdot \frac{\partial \boldsymbol{\varepsilon}}{\partial \boldsymbol{\mu}}\right\} \cdot \frac{\partial \boldsymbol{\mu}}{\partial t} \mathrm{~d} \mathbf{x} \\
& =\int_{\Omega}-\mathbf{F} \cdot \frac{\partial \boldsymbol{\mu}}{\partial t} \mathrm{~d} \mathbf{x}=-M \int_{\Omega}|\mathbf{F}|^{2} \mathrm{~d} \mathbf{x} \leqslant 0
\end{aligned}
$$

due to Eq. (22), $M>0$ and the arguments similar to those used to derive $\delta \mathscr{I}(\boldsymbol{\mu})$ in Eq. (18). As the result of Eq. (23), Eq. (22) will be used as our fundamental tool to investigate the formation of martensitic patterns under different conditions.

\subsection{Thin film limit}

Consider a single crystal film of thickness $h$. Suppose it is released on a certain region $S \subset \mathbb{R}^{2}$ but is attached to a substrate outside it, as shown in the left of Fig. 3. Very often the thickness of the film is much smaller than the lateral extent. In this situation, Bhattacharya and James [5] have shown that the out-of-plane strain incompatibility can be neglected. Therefore, only the in-plane components of the transformation strains need to be considered. As a result, the criterion for coherence is weakened in thin films and may depend on the film normals. To be precise, let $\Pi$ be the $2 \times 3$ matrix

$$
\Pi=\left(\begin{array}{lll}
1 & 0 & 0 \\
0 & 1 & 0
\end{array}\right)
$$

and $\mathbf{R}$ be the proper rotation associated with the film normal. The in-plane transformation strain field $\varepsilon_{p}^{*}(\boldsymbol{\mu})$ in this case is defined by

$\boldsymbol{\varepsilon}_{p}^{*}(\boldsymbol{\mu})=\sum_{i=1}^{N} \gamma_{i}(\boldsymbol{\mu}) \boldsymbol{\varepsilon}_{p}^{(i)}, \quad \boldsymbol{\varepsilon}_{p}^{(i)}=\Pi\left(\mathbf{R} \boldsymbol{\varepsilon}^{(i)} \mathbf{R}^{\mathrm{T}}\right) \Pi^{\mathrm{T}}$
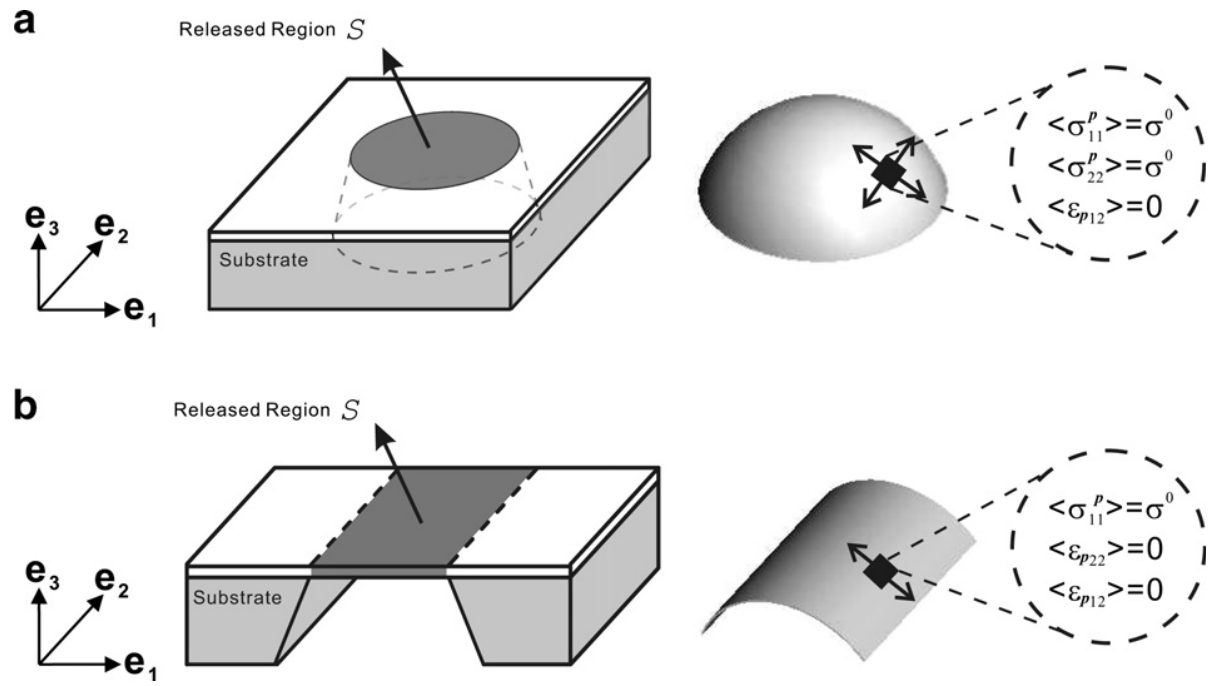

Fig. 3. Two prototypes of microactuators with dome-shaped and tunnel-shaped deformations depicted in (a) and (b), respectively. 
where $\gamma_{i}(\boldsymbol{\mu})$ is given by Eq. (5) and $\boldsymbol{\varepsilon}_{p}^{(i)}$ is the in-plane transformation strain of the $i$ th martensitic variant associated with the orientation $\mathbf{R}$. According to the theory proposed by Bhattacharya and James [5] and Shu [6,43], the problem can be reduced to a two-dimensional setting since the energy consists of two contributions: the one scales at an order of $h$ and the other has an $h^{3}$ scaling, i.e.

$$
\begin{aligned}
& \mathscr{I}(\boldsymbol{\mu})=h \int_{S}\left\{W_{p}^{\mathrm{int}}+W_{p}^{\mathrm{ani}}(\boldsymbol{\mu})+W_{p}^{\mathrm{elas}}(\boldsymbol{\mu})-\boldsymbol{\sigma}^{p 0} \cdot \boldsymbol{\varepsilon}_{p}\right\} \mathrm{d} \mathbf{x}_{p}+\mathrm{O}\left(h^{3}\right) \\
& W_{p}^{\mathrm{int}}(\boldsymbol{\mu})=A\left|\nabla_{p} \boldsymbol{\mu}\right|^{2}, W_{p}^{\mathrm{ani}}(\boldsymbol{\mu})=W^{\mathrm{ani}}(\boldsymbol{\mu}), \\
& W_{p}^{\text {elas }}(\boldsymbol{\mu})=\frac{1}{2}\left[\boldsymbol{\varepsilon}_{p}-\boldsymbol{\varepsilon}_{p}^{*}(\boldsymbol{\mu})\right] \cdot \mathbf{C}^{p}\left[\boldsymbol{\varepsilon}_{p}-\boldsymbol{\varepsilon}_{p}^{*}(\boldsymbol{\mu})\right]
\end{aligned}
$$

where $\nabla_{p}$ is the in-plane gradient with respect to the planar variables $\mathbf{x}_{p}=\left(x_{1}, x_{2}\right) \in S, \boldsymbol{\varepsilon}_{p}$ is the in-plane strain, $\boldsymbol{\sigma}^{p 0}$ is the in-plane auxiliary stress state, which is divergence-free and consistent with the applied traction at the boundary, and $\mathbf{C}^{p}$ is the conventional plane-stress elastic modulus. The term $\mathrm{O}\left(h^{3}\right)$ is the energy scaling on the order of $h^{3}$. Thus, at the first-order approximation, the evolution of microstructure is governed by

$$
\begin{aligned}
\frac{\partial \boldsymbol{\mu}}{\partial t} & =M\left(\mathbf{F}_{p}^{\mathrm{int}}+\mathbf{F}_{p}^{\text {ani }}+\mathbf{F}_{p}^{\text {elas }}\right) \\
\mathbf{F}_{p}^{\text {int }} & =2 A \nabla_{p}^{2} \boldsymbol{\mu}, \quad \mathbf{F}_{p}^{\text {ani }}=\mathbf{F}^{\text {ani }}, \quad \mathbf{F}_{p}^{\text {elas }}=\mathbf{C}^{p}\left[\boldsymbol{\varepsilon}_{p}-\boldsymbol{\varepsilon}_{p}^{*}(\boldsymbol{\mu})\right] \cdot \frac{\partial \boldsymbol{\varepsilon}_{p}^{*}(\boldsymbol{\mu})}{\partial \boldsymbol{\mu}}
\end{aligned}
$$

under the elastic constraint

$$
\nabla_{p} \cdot \boldsymbol{\sigma}^{p}=0, \quad \boldsymbol{\sigma}^{p}=\mathbf{C}^{p}\left[\boldsymbol{\varepsilon}_{p}-\boldsymbol{\varepsilon}_{p}^{*}(\boldsymbol{\mu})\right]
$$

As the length scale of martensitic microstructure is typically much smaller than the lateral boundary size of the film, it is reasonable to assume the periodic boundary condition in Eq. (26), i.e. $\mu$ is periodic on a small square $\left(0, l_{0}\right)^{2}$. In addition, the in-plane stress $\boldsymbol{\sigma}^{p}$ can be decomposed as the sum of the homogeneous $\left\langle\boldsymbol{\sigma}^{p}\right\rangle$ and perturbed $\boldsymbol{\sigma}^{p \prime}$ states, as discussed in Eq. (14) and Eq. (15). The perturbed inhomogeneous stress $\boldsymbol{\sigma}^{p \prime}$ can be explicitly obtained in the Fourier reciprocal space, as provided by Eq. (A3). The homogeneous in-plane stress $\left\langle\sigma^{p}\right\rangle$, however, depends on the imposed boundary conditions, such as specifying the overall strain, as in Eq. (16), or the overall stress, as in Eq. (17).

Finally, the development of various kinds of microactuators motivates the consideration of the mixed types of boundary conditions. For example, suppose the released region $S$ of the film, shown in the left of Fig. 3a, is a circle. It is expected to obtain a dome-shaped deformation under perhaps some back pressure. Thus, a small element of the film, taken out away from the lateral boundary, may sustain $\left\langle\boldsymbol{\sigma}_{11}^{p}\right\rangle=\boldsymbol{\sigma}^{0}>0,\left\langle\boldsymbol{\sigma}_{22}^{p}\right\rangle=\boldsymbol{\sigma}^{0}>0$ and $\left\langle\boldsymbol{\varepsilon}_{p_{12}}\right\rangle=0$, as illustrated in the right of Fig. 3a. The tensile stress $\sigma^{0}>0$ is induced due to some back pressure, while its magnitude may be different at distinct locations. Under these circumstances, the elastic stress in this case is

$$
\begin{aligned}
\boldsymbol{\sigma}_{11}^{p} & =\boldsymbol{\sigma}^{0}+\boldsymbol{\sigma}_{11}^{p \prime} \\
\boldsymbol{\sigma}_{22}^{p} & =\boldsymbol{\sigma}^{0}+\boldsymbol{\sigma}_{22}^{p \prime} \\
\boldsymbol{\sigma}_{12}^{p} & =\frac{\boldsymbol{\sigma}^{0}}{C_{11}^{p} C_{22}^{p}-\left(C_{12}^{p}\right)^{2}}\left[C_{22}^{p} C_{16}^{p}+C_{11}^{p} C_{26}^{p}-C_{12}^{p}\left(C_{16}^{p}+C_{26}^{p}\right)\right] \\
& +\frac{2\left\langle\boldsymbol{\varepsilon}_{p_{12}}^{*}\right\rangle}{C_{11}^{p} C_{22}^{p}-\left(C_{12}^{p}\right)^{2}}\left[C_{22}^{p}\left(C_{16}^{p}\right)^{2}+C_{11}^{p}\left(C_{26}^{p}\right)^{2}-2 C_{12}^{p} C_{16}^{p} C_{26}^{p}\right] \\
& -2 C_{66}^{p}\left\langle\boldsymbol{\varepsilon}_{p_{12}}^{*}\right\rangle+\boldsymbol{\sigma}_{12}^{p \prime}
\end{aligned}
$$

where $C_{i j}^{p}$ is the plane-stress elastic constant in terms of Voigt notation $(i, j=1,2,6)$. Another common example is that the released region $S$ of the film is a strip rather than a circle, as shown in the left of Fig. 3b. A tunnel-shaped deformation is anticipated. Since two parts of the film are attached to a substrate, this indicates that the film suffers no shear in the strip when it transforms to martensite, and is stretched in a direction perpendicular to the strip. Hence, if a small portion of the film is taken out and examined, as shown in the right of Fig. 3b, it sustains a tensile stress $\left\langle\boldsymbol{\sigma}_{11}^{p}\right\rangle=\boldsymbol{\sigma}^{0}>0$ with $\left\langle\boldsymbol{\varepsilon}_{p_{12}}\right\rangle=0$ and $\left\langle\boldsymbol{\varepsilon}_{p_{22}}\right\rangle=0$ to ensure a tunnel-like configuration. In this case, the elastic stress is

$$
\begin{aligned}
\boldsymbol{\sigma}_{11}^{p}= & \boldsymbol{\sigma}^{0}+\boldsymbol{\sigma}_{11}^{p \prime} \\
\boldsymbol{\sigma}_{22}^{p}= & \frac{C_{12}^{p}}{C_{11}^{p}} \boldsymbol{\sigma}^{0}+\left(\frac{C_{12}^{p} C_{12}^{p}}{C_{11}^{p}}-C_{22}^{p}\right)\left\langle\varepsilon_{p_{22}}^{*}\right\rangle \\
& +2\left(\frac{C_{12}^{p} C_{16}^{p}}{C_{11}^{p}}-C_{26}^{p}\right)\left\langle\varepsilon_{p_{12}}^{*}\right\rangle+\boldsymbol{\sigma}_{22}^{p \prime} \\
\boldsymbol{\sigma}_{12}^{p}= & \frac{C_{16}^{p}}{C_{11}^{p}} \boldsymbol{\sigma}^{0}+\left(\frac{C_{12}^{p} C_{16}^{p}}{C_{11}^{p}}-C_{26}^{p}\right)\left\langle\varepsilon_{p_{22}}^{*}\right\rangle \\
& +2\left(\frac{C_{16}^{p} C_{16}^{p}}{C_{11}^{p}}-C_{66}^{p}\right)\left\langle\varepsilon_{p_{12}}^{*}\right\rangle+\boldsymbol{\sigma}_{12}^{p \prime}
\end{aligned}
$$

\subsection{Numerical implementation}

It is common to normalize the length and time scales in Eq. (26) under the periodic boundary condition. Indeed, set

$\tilde{x}_{1}=\frac{x_{1}}{l_{0}}, \quad \tilde{x}_{2}=\frac{x_{2}}{l_{0}}, \quad \tilde{t}=2 M K t$

where $l_{0}$ is the size of the simulation. The evolution of martensitic microstructure in thin films governed by Eq. (26) can be normalized to be

$\frac{\partial \boldsymbol{\mu}}{\partial \tilde{t}}=D \tilde{\nabla}_{p}^{2} \boldsymbol{\mu}+\frac{1}{2 K}\left[\mathbf{F}_{p}^{\text {ani }}(\boldsymbol{\mu})+\mathbf{F}_{p}^{\text {elas }}(\boldsymbol{\mu})\right], \quad\left(\tilde{x}_{1}, \tilde{x}_{2}\right) \in(0,1)^{2}$

where $\tilde{\nabla}_{p}^{2}=\frac{\partial^{2}}{\partial \tilde{x}_{1}^{2}}+\frac{\partial^{2}}{\partial \tilde{x}_{2}^{2}}$ and $D=\frac{A / K}{l_{0}^{2}}$. The dimensionless parameter $\sqrt{D}$ is related to the length scale of the interface.

Solving Eq. (31) by an explicit forward Euler scheme is not efficient since it requires very small time steps to maintain stability. On the other hand, the implicit backward Euler method is also not suitable since $\mathbf{F}_{p}^{\text {ani }}(\boldsymbol{\mu})$ and $\mathbf{F}_{p}^{\text {elas }}(\boldsymbol{\mu})$ are nonlinear in $\boldsymbol{\mu}$ in general, causing nonlinear 
equations to be solved at each time step. To compromise, the semi-implicit method proposed by Chen and Shen [44] is chosen to solve Eq. (31). This scheme treats the Laplacian operator implicitly to relax the stability constraint, while the nonlinear terms are specified explicitly to avoid solving nonlinear algebraic equations at each iteration. Specifically, let $\Delta \tilde{t}$ be the small time step, $\boldsymbol{\mu}^{n}=$ $\boldsymbol{\mu}\left(\tilde{x}_{1}, \tilde{x}_{2}, n \Delta \tilde{t}\right)$ and $\boldsymbol{\mu}^{n+1}=\boldsymbol{\mu}\left(\tilde{x}_{1}, \tilde{x}_{2},(n+1) \Delta \tilde{t}\right)$. Applying the semi-implicit finite difference scheme to Eq. (31) provides

$\frac{\boldsymbol{\mu}^{n+1}-\boldsymbol{\mu}^{n}}{\Delta \tilde{t}}=D \tilde{\nabla}_{p}^{2} \boldsymbol{\mu}^{n+1}+\frac{1}{2 K}\left[\mathbf{F}_{p}^{\text {ani }}\left(\boldsymbol{\mu}^{n}\right)+\mathbf{F}_{p}^{\text {elas }}\left(\boldsymbol{\mu}^{n}\right)\right]$

Under the periodic boundary condition, it is convenient to solve Eq. (32) in the Fourier space. Taking the Fourier transform on both sides of Eq. (32) gives

$\overline{\boldsymbol{\mu}}^{n+1}=\frac{1}{\left\{1+4 \pi^{2} D\left(\xi_{1}^{2}+\xi_{2}^{2}\right) \Delta \tilde{t}\right\}}\left\{\overline{\boldsymbol{\mu}}^{n}+\frac{\Delta \tilde{t}}{2 K}\left[\overline{\mathbf{F}_{p}^{\text {ani }}\left(\boldsymbol{\mu}^{n}\right)}+\overline{\mathbf{F}_{p}^{\text {elas }}\left(\boldsymbol{\mu}^{n}\right)}\right]\right\}$

where $\xi_{1}$ and $\xi_{2}$ are coordinates in the reciprocal space, $\overline{\boldsymbol{\mu}^{n+1}}=\mathscr{F}\left[\boldsymbol{\mu}^{n+1}\right], \overline{\boldsymbol{\mu}^{n}}=\mathscr{F}\left[\boldsymbol{\mu}^{n}\right], \overline{\mathbf{F}_{p}^{\text {ani }}(\boldsymbol{\mu})}=\mathscr{F}\left[\mathbf{F}_{p}^{\text {ani }}(\boldsymbol{\mu})\right], \overline{\mathbf{F}_{p}^{\text {elas }}(\boldsymbol{\mu})}=$ $\mathscr{F}\left[\mathbf{F}_{p}^{\text {elas }}(\boldsymbol{\mu})\right]$ and the Fourier operator $\mathscr{F}[\cdot]$ is defined by Eq. (A1). The variable $\mu^{n+1}$ in the real space can be obtained through an inverse Fourier transform of $\overline{\boldsymbol{\mu}^{n+1}}$ defined by Eq. (A2).

\section{Simulation results}

Now the various types of patterns in martensitic thin films are provided here. The chosen material for simulation is $\mathrm{Ti}-\mathrm{Ni}$ at the trigonal $\mathrm{R}$-phase. Thus, the number of martensitic variants is $N=4$, and from [40], $\alpha=0, \delta=0.0047$ in Eq. (2). The elastic moduli of Ti-Ni single crystals are not available; therefore, we take $C_{11}^{p}=C_{22}^{p}=80 \mathrm{GPa}$, $C_{12}^{p}=20 \mathrm{GPa}, C_{66}^{p}=30 \mathrm{GPa}$ and $C_{16}^{p}=C_{26}^{p}=0$ in Eq. (A3). They are typical parameters for Ti-Ni polycrystals [45]. Besides, there are two dimensionless parameters in the evolution Eq. (31). The first one, $D=\frac{A / K}{l_{0}^{2}}$, related to the length scale of microstructure, is taken to be $D=$ 0.0001 [18]. Another dimensionless parameter is related to the ratio of elastic energy to anisotropy energy. In the present simulation, $K$ is chosen such that $\frac{\varepsilon_{p}^{(1)} \cdot \mathbf{C}^{p} \varepsilon_{p}^{(1)}}{K}=1$, where $\boldsymbol{\varepsilon}_{p}^{(1)}$ is given by Eq. (35). Thus, the energy densities $W_{p}^{\text {ani }}$ and $W_{p}^{\text {elas }}$ are of the same order. As described in Section 2.4, the criterion for compatibility in thin films depends on film normals. We consider three common crystallographic orientations: (001), (110) and (111) films, given by

$$
\begin{aligned}
\mathbf{R}_{(001)} & =\left(\begin{array}{ccc}
\frac{1}{\sqrt{2}} & \frac{1}{\sqrt{2}} & 0 \\
\frac{-1}{\sqrt{2}} & \frac{1}{\sqrt{2}} & 0 \\
0 & 0 & 1
\end{array}\right), \quad \mathbf{R}_{(110)}=\left(\begin{array}{ccc}
\frac{1}{\sqrt{2}} & \frac{1}{\sqrt{2}} & 0 \\
0 & 0 & 1 \\
\frac{1}{\sqrt{2}} & \frac{-1}{\sqrt{2}} & 0
\end{array}\right), \\
\mathbf{R}_{(111)} & =\left(\begin{array}{ccc}
\frac{1}{\sqrt{2}} & -\frac{1}{\sqrt{2}} & 0 \\
\frac{1}{\sqrt{6}} & \frac{1}{\sqrt{6}} & \frac{-2}{\sqrt{6}} \\
\frac{1}{\sqrt{3}} & \frac{1}{\sqrt{3}} & \frac{1}{\sqrt{3}}
\end{array}\right)
\end{aligned}
$$

in Eq. (24). A variety of periodic boundary conditions are taken for simulations, and the fast Fourier transform is employed to enhance the speed of computation. Since the nucleation problem, while important in general, is not the central issue in the present study, we take the random initial conditions for all simulations.

\section{1. (001) Film}

According to Eq. (24), the in-plane transformation strains under the rotation $\mathbf{R}_{(001)}$ given by Eq. (34) are

$$
\boldsymbol{\varepsilon}_{p}^{(1)}=\boldsymbol{\varepsilon}_{p}^{(4)}=\left(\begin{array}{cc}
\alpha+\delta & 0 \\
0 & \alpha-\delta
\end{array}\right), \quad \boldsymbol{\varepsilon}_{p}^{(2)}=\boldsymbol{\varepsilon}_{p}^{(3)}=\left(\begin{array}{cc}
\alpha-\delta & 0 \\
0 & \alpha+\delta
\end{array}\right)
$$

Thus, there are only two distinct variants. Suppose the film is unstressed as deposited, and is partially released in a chosen region $S$. As the film is still attached to the substrate in the surrounding region, as illustrated in the left of Fig. 3a, it is reasonable to assume the clamped boundary condition, i.e. $\left\langle\boldsymbol{\varepsilon}_{p}\right\rangle=\mathbf{0}$. The simulation result shows that the only selfaccommodation pattern is the lamellar type, as shown in Fig. 4a. Notice that different variants are presented by different gray levels, as listed at the bottom of Fig. 2. The interface separating variants (1) and (3) is compatible since $\boldsymbol{\varepsilon}_{p}^{(1)}-\boldsymbol{\varepsilon}_{p}^{(3)}=\frac{1}{2}\left(\mathbf{a}_{13} \otimes \mathbf{n}_{13}+\mathbf{n}_{13} \otimes \mathbf{a}_{13}\right)$ and $\mathbf{n}_{13}=\frac{1}{\sqrt{2}}(1,1)$ or $\mathbf{n}_{13}=\frac{1}{\sqrt{2}}(-1,1)$, as can be seen in the right of Fig. 4a.

Finally, the lamellar pattern is not a basic unit for selfaccommodation in bulk trigonal martensite [46], since the average of the transformation strains does not vanish, i.e. $0.5 \boldsymbol{\varepsilon}^{(1)}+0.5 \boldsymbol{\varepsilon}^{(3)} \neq \mathbf{0}$. However, this pattern in thin films is self-accommodated due to the zero average of the in-plane transformation strains, i.e. $0.5 \boldsymbol{\varepsilon}_{p}^{(1)}+0.5 \boldsymbol{\varepsilon}_{p}^{(3)}=\mathbf{0}$. As a result, the pattern shown in Fig. 4a is observed often in many (001) films with trigonal lattices [47].

\section{2. (110) Film}

According to Eq. (24), the in-plane transformation strains under the rotation $\mathbf{R}_{(110)}$ given by Eq. (34) are

$$
\begin{aligned}
& \boldsymbol{\varepsilon}_{p}^{(1)}=\left(\begin{array}{cc}
\alpha+\delta & \sqrt{2} \delta \\
\sqrt{2} \delta & \alpha
\end{array}\right), \quad \boldsymbol{\varepsilon}_{p}^{(2)}=\boldsymbol{\varepsilon}_{p}^{(3)}=\left(\begin{array}{cc}
\alpha-\delta & 0 \\
0 & \alpha
\end{array}\right), \\
& \boldsymbol{\varepsilon}_{p}^{(4)}=\left(\begin{array}{cc}
\alpha+\delta & -\sqrt{2} \delta \\
-\sqrt{2} \delta & \alpha
\end{array}\right)
\end{aligned}
$$

From Eq. (36), variants (2) and (3) are indistinguishable according to the Bhattacharya-James thin film theory [5], and therefore there are three distinct variants in this case. Under the clamped boundary condition, $\left\langle\boldsymbol{\varepsilon}_{p}\right\rangle=\mathbf{0}$, two different kinds of self-accommodation patterns are predicted from the simulations. The first one, shown in Fig. $4 b$, is similar to the herring-bone pattern commonly observed in trigonal martensites [46]. Another pattern which is much simpler than the previous one is shown in Fig. 4c. Notice that it is not an allowable pattern in bulk martensites, since 
a

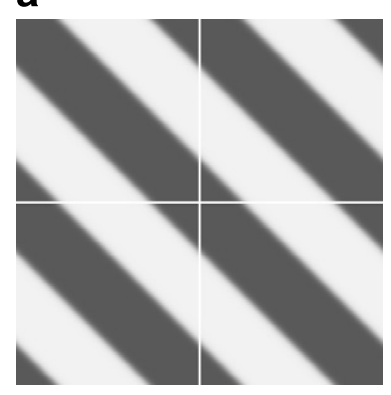

b

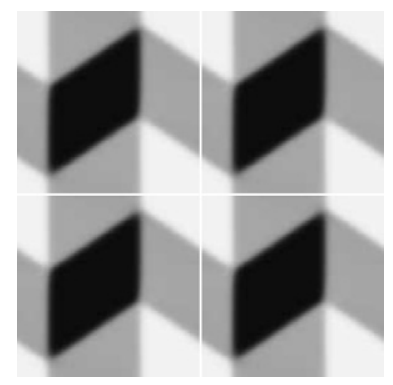

C
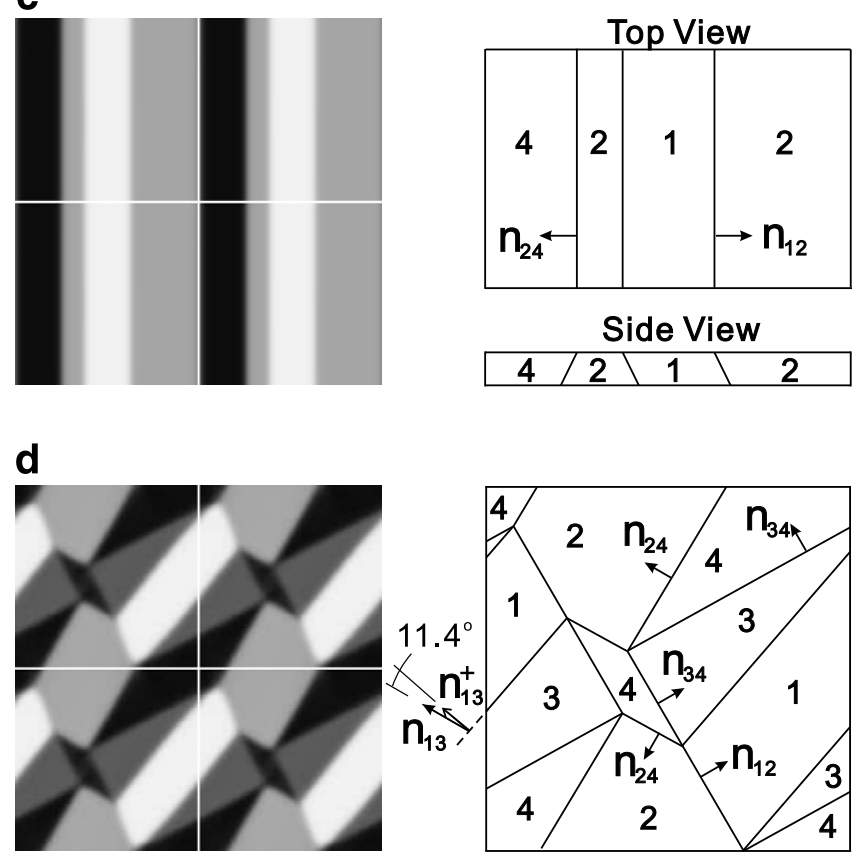

Fig. 4. Self-accommodation patterns for various film orientations: (a) for (001) films; (b) and (c) for (110) films; and (d) for (111) films. All of the interfaces are compatible except those with normals $\mathbf{n}_{13}^{+}$in (d). Notice that four identical patterns are packed together to obtain a better image.

the third components in the interfacial normals are different, as can be seen in the right of Fig. 4c. However, it is a legitimate one in thin films, and this confirms that martensitic materials can form many more interfaces in thin films than in bulk. In addition, the self-accommodation requires $\gamma_{1} \varepsilon_{p}^{(1)}+\gamma_{2} \varepsilon_{p}^{(2)}+\gamma_{4} \varepsilon_{p}^{(4)}=\mathbf{0}$, giving rise to $\gamma_{1}=0.25$, $\gamma_{2}=0.5, \gamma_{4}=0.25$. The simulated volume fractions are $\gamma_{1}=0.248, \gamma_{2}=0.502, \gamma_{4}=0.25$ in Fig. $4 b$, and $\gamma_{1}=0.25$,
Table 2

Compatible interfacial normals in (110) films

\begin{tabular}{lclc}
\hline Variants & 1,2 or 1,3 & 1,4 & 2,4 or 3,4 \\
\hline$\{100\}$ type & $(1,0)$ & $(0,1)$ & $(1,0)$ \\
$\{110\}$ type & $(1, \sqrt{2})$ & $(1,0)$ & $(-1, \sqrt{2})$ \\
\hline
\end{tabular}

$\gamma_{2}=0.5, \gamma_{4}=0.25$ in Fig. 4c. They are all consistent with the theoretical prediction.

Finally, Table 2 contains all possible compatible interfaces in (110) films predicted based on the thin film compatibility. For example,

$\boldsymbol{\varepsilon}_{p}^{(1)}-\boldsymbol{\varepsilon}_{p}^{(2)}=\delta\left(\begin{array}{l}1 \\ 0\end{array}\right) \otimes\left(\begin{array}{c}1 \\ \sqrt{2}\end{array}\right)+\delta\left(\begin{array}{c}1 \\ \sqrt{2}\end{array}\right) \otimes\left(\begin{array}{l}1 \\ 0\end{array}\right)$

Thus, $(1,0)$ and $(1, \sqrt{2})$ are the only two compatible interfacial normals separating variants (1) and (2). The simulation results confirm that all the interfacial normals in Fig. $4 \mathrm{~b}$ and $\mathrm{c}$ agree very well with those listed in Table 2.

\section{3. (11 11 ) Film}

According to Eq. (24), the in-plane transformation strains under the rotation $\mathbf{R}_{(111)}$ given by Eq. (34) are

$$
\begin{aligned}
\boldsymbol{\varepsilon}_{p}^{(1)}=\left(\begin{array}{cc}
\alpha-\delta & 0 \\
0 & \alpha-\delta
\end{array}\right) & \boldsymbol{\varepsilon}_{p}^{(2)}=\left(\begin{array}{cc}
\alpha+\delta & \frac{2}{\sqrt{3}} \delta \\
\frac{2}{\sqrt{3}} \delta & \alpha-\frac{1}{3} \delta
\end{array}\right) \\
\boldsymbol{\varepsilon}_{p}^{(3)}=\left(\begin{array}{cc}
\alpha+\delta & \frac{-2}{\sqrt{3}} \delta \\
\frac{-2}{\sqrt{3}} \delta & \alpha-\frac{1}{3} \delta
\end{array}\right) & \boldsymbol{\varepsilon}_{p}^{(4)}=\left(\begin{array}{cc}
\alpha-\delta & 0 \\
0 & \alpha+\frac{5}{3} \delta
\end{array}\right)
\end{aligned}
$$

They are all different in this case, and a self-accommodation pattern is expected to contain all four of these martensitic variants with equal volume fractions, since $0.25 \varepsilon_{p}^{(1)}+0.25 \varepsilon_{p}^{(2)}+0.25 \varepsilon_{p}^{(3)}+0.25 \varepsilon_{p}^{(4)}=\mathbf{0}$. Indeed, the simulated pattern shown in Fig. 4d under the clamped boundary condition gives the volume fractions $\gamma_{1}=0.247$, $\gamma_{2}=0.250, \gamma_{3}=0.252$, and $\gamma_{4}=0.251$. Each of them is close to the theoretical prediction. However, certain interfacial normals deviate from those listed in Table 3 which contains all possible compatible interfaces in (111) films. Precisely,

$\boldsymbol{\varepsilon}_{p}^{(1)}-\boldsymbol{\varepsilon}_{p}^{(3)}=\frac{-\delta}{3}\left\{\left(\begin{array}{c}-\sqrt{3} \\ 1\end{array}\right) \otimes\left(\begin{array}{c}-\sqrt{3} \\ 1\end{array}\right)+\left(\begin{array}{c}-\sqrt{3} \\ 1\end{array}\right) \otimes\left(\begin{array}{c}-\sqrt{3} \\ 1\end{array}\right)\right\}$

Thus, $\mathbf{n}_{13}=(-\sqrt{3}, 1)$ is the only compatible normal direction separating variants (1) and (3). However, all of the interfacial normals shown in Fig. $4 \mathrm{~d}$ are found to be in good agreement with those listed in Table 3 except $\mathbf{n}_{13}^{+}$. A further investigation reveals that the discrepancy between $\mathbf{n}_{13}$ and $\mathbf{n}_{13}^{+}$is around $11.4^{\circ}$. It indicates that Fig. $4 d$ does not reach a minimum energy solution with average excess stress fields $\left\langle\left|\boldsymbol{\sigma}_{11}^{p}\right|\right\rangle=6.4 \times 10^{-5} C_{12}^{p},\left\langle\left|\boldsymbol{\sigma}_{22}^{p}\right|\right\rangle=13.9 \times 10^{-5} C_{12}^{p}$ and $\left\langle\left|\boldsymbol{\sigma}_{12}^{p}\right|\right\rangle=5.7 \times 10^{-5} C_{12}^{p}$, respectively. 
Table 3

Compatible interfacial normals in (111) films

\begin{tabular}{lllllll}
\hline Variants & 1,2 & 1,3 & 1,4 & 2,3 & 2,4 & 3,4 \\
\hline$\{100\}$ type & $(\sqrt{3}, 1)$ & $(-\sqrt{3}, 1)$ & $(0,1)$ & $(0,1)$ & $(-\sqrt{3}, 1)$ & $(\sqrt{3}, 1)$ \\
$\{110\}$ type & $(\sqrt{3}, 1)$ & $(-\sqrt{3}, 1)$ & $(0,1)$ & $(1,0)$ & $(1, \sqrt{3})$ & $(1,-\sqrt{3})$ \\
\hline
\end{tabular}

\subsection{Design of large strain microactuators}

We now apply the present framework to the design of large strain microactuators with dome-shaped and tunnel-shaped configurations. Suppose the film is deposited on a substrate first, and is released in some chosen region $S$ by etching. It is flat and taut at high temperatures, while it bulges up in the martensite phase at low temperatures, under perhaps some back pressure. Thus, the released portion of the film swells out and pulls back, functioning as an actuator by thermal cycling. The task here is to determine the optimal orientation and microstructure of the film to maximize the deformed volume.

Suppose the released region $S$ of film, shown in the left of Fig. 3a, is a circle. It is expected to bulge out like a dome in the martensite phase. As discussed in Section 2.4, the film sustains a biaxial stress due to some back pressure such that $\left\langle\boldsymbol{\sigma}_{11}^{p}\right\rangle=\boldsymbol{\sigma}^{0},\left\langle\boldsymbol{\sigma}_{22}^{p}\right\rangle=\boldsymbol{\sigma}^{0}$ and $\left\langle\boldsymbol{\varepsilon}_{p_{12}}\right\rangle=0$, as demonstrated in the right of Fig. 3a. To obtain large pumping volume, we seek for the optimal microstructure such that the trace of $\left\langle\boldsymbol{\varepsilon}_{p}\right\rangle,\left\langle\boldsymbol{\varepsilon}_{p_{11}}+\boldsymbol{\varepsilon}_{p_{22}}\right\rangle$, is maximized [8]. It can be determined using Eq. (26) for microstructure simulation and Eq. (28) for stress computation under $\sigma^{0}=5 \mathrm{MPa}$. The resulting patterns of microstructure are shown in Fig. 5 for (110) and (111) films. The pattern for (001) films is similar to the self-accommodated pattern shown in Fig. 4a due to symmetry. Thus, it is not shown here. To explain these stressed patterns for (1110) and (111) films, an energy argument is employed. The auxiliary stress state is $\boldsymbol{\sigma}^{p 0}=\boldsymbol{\sigma}^{0} \mathbf{I}$ in Eq. (25), where I is the identity tensor. For (110) films, the potential energy due to this biaxial stress is positive for variant (2): $-\left(\boldsymbol{\sigma}^{0} \mathbf{I} \cdot \boldsymbol{\varepsilon}_{p}^{(2)}\right)=\delta \boldsymbol{\sigma}^{0}>0$. However, it is negative and identical for both variants (1) and (4): $-\left(\boldsymbol{\sigma}^{0} \mathbf{I} \cdot \boldsymbol{\varepsilon}_{p}^{(1)}\right)=$ $-\left(\boldsymbol{\sigma}^{0} \mathbf{I} \cdot \boldsymbol{\varepsilon}_{p}^{(4)}\right)=-\delta \boldsymbol{\sigma}^{0}<0$. Thus, variant (2) disappears in Fig. 5a. Moreover, from Table 2, the only compatible interface separating variants (1) and (4) is $\mathbf{n}_{14}=(1,0)$ or $\mathbf{n}_{14}=(0,1)$, giving rise to the final pattern shown in Fig. 5a. Next, applying this energy argument to the stressed (111) film gives

$$
\begin{aligned}
& -\left(\boldsymbol{\sigma}^{0} \mathbf{I} \cdot \boldsymbol{\varepsilon}_{p}^{(1)}\right)=2 \delta \boldsymbol{\sigma}^{0}>0, \\
& -\left(\boldsymbol{\sigma}^{0} \mathbf{I} \cdot \boldsymbol{\varepsilon}_{p}^{(2)}\right)=-\left(\boldsymbol{\sigma}^{0} \mathbf{I} \cdot \boldsymbol{\varepsilon}_{p}^{(3)}\right)=-\left(\boldsymbol{\sigma}^{0} \mathbf{I} \cdot \boldsymbol{\varepsilon}_{p}^{(4)}\right)=-\frac{2}{3} \delta \boldsymbol{\sigma}^{0}<0
\end{aligned}
$$

Thus, variant (1) vanishes and the other variants are energetically equally favorable, leading to a pattern shown in Fig. 5b. Finally, Table 4 lists the sum of the principal strains for these patterns. It shows that (110) films provides the largest biaxial stretch under the same stress state.

We now turn to another case where the released portion of the film is a strip, as shown in the left of Fig. 3b. As described in Section 2.4, to ensure a tunnel-like configuration, it requires $\left\langle\boldsymbol{\sigma}_{11}^{p}\right\rangle=\boldsymbol{\sigma}^{0}>0$ with $\left\langle\boldsymbol{\varepsilon}_{p_{22}}\right\rangle=0$ and $\left\langle\boldsymbol{\varepsilon}_{p_{12}}\right\rangle=0$ in any small element of the film taken away from the boundary constraints, as illustrated in the right of Fig. 3b. Now the task here is to look for the microstructure such that the tensile strain $\left\langle\varepsilon_{p_{11}}\right\rangle$ is maximized for different orientations of the film. The elastic stress field is computed using Eq. (29) under $\boldsymbol{\sigma}^{0}=5 \mathrm{MPa}$, and the simulation results via Eq. (26) are shown in Fig. 6a for (110) films and Fig. 6b for (111) films. The pattern for (001) films is similar to that in Fig. 4a, therefore, is not shown here. Fig. 6 can be understood in a way similar to the previous case by comparing the potential energy of this uniaxial stress for each of the variants. The results show that variants (1) and (4) are favorable for (110) film. This gives the pattern shown in Fig. 6a since, from Table 2, the only

Table 4

The average strains for different orientations of the film in the cases of dome-shaped and tunnel-shaped deformations

\begin{tabular}{llll}
\hline Types of microactuators & $(001)$ film & $(110)$ film & $(111)$ film \\
\hline Dome: $\left\langle\varepsilon_{p_{11}}+\varepsilon_{p_{22}}\right\rangle(\%)$ & $\approx 0$ & 0.48 & 0.34 \\
$x_{2}$-tunnel: $\left\langle\varepsilon_{p_{11}}\right\rangle(\%)$ & $\approx 0$ & 0.48 & 0.34 \\
$x_{1}$-tunnel: $\left\langle\varepsilon_{p_{22}}\right\rangle(\%)$ & $\approx 0$ & $\approx 0$ & 0.32 \\
\hline
\end{tabular}

Here, " $x_{2}$-tunnel" denotes that the orientation of a tunnel is along the $x_{2^{-}}$ direction (see Fig. 3b), while " $x_{1}$-tunnel" is along the $x_{1}$-direction.
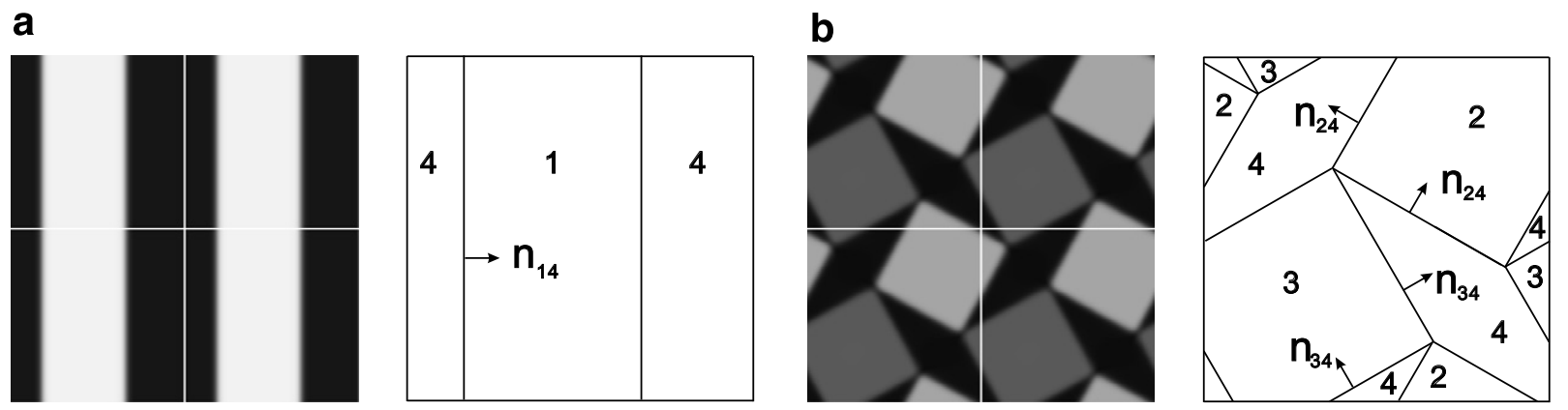

Fig. 5. Patterns of microstructure in dome-shaped deformations: (a) for (1 110$)$ films and (b) for (111) films. Note that these two stressed patterns remain compatible during evolution. 
a

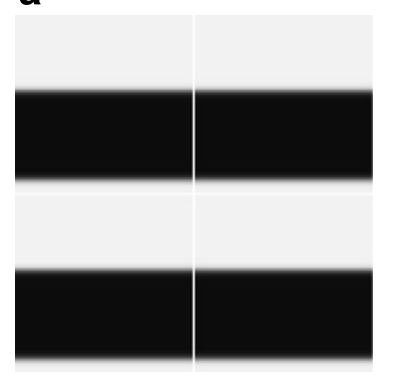

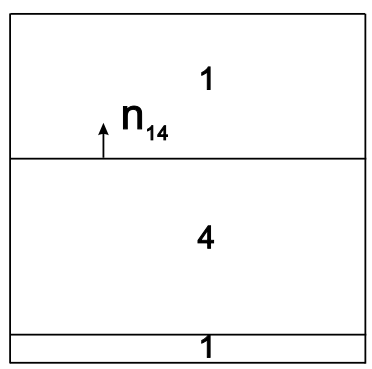

b

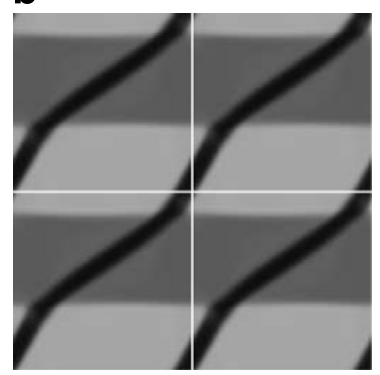

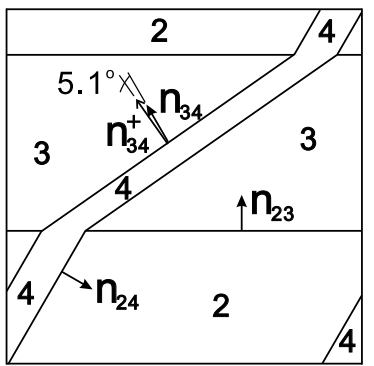

Fig. 6. Patterns of microstructure in tunnel-shaped deformations: (a) for (1 110$)$ films and (b) for (1 111 ) films. The orientation of the tunnel is along the $x_{2}$ direction, as shown in Fig. 3b. All of the interfaces are coherent except $\mathbf{n}_{34}^{+}$, which deviates from $\mathbf{n}_{34}$ by only $5.1^{\circ}$.

compatible interfacial normals between variants (1) and (4) in (1 110$)$ films are either $\mathbf{n}_{14}=(1,0)$ or $\mathbf{n}_{14}=(0,1)$. Next, it can also be shown that variants (2) and (3) are energetically favorable for (111) films under the uniaxial stretch. However, in the pattern shown in Fig. 6b there appears a small fraction of the unfavorable variant (4) due to the requirement of $\left\langle\boldsymbol{\varepsilon}_{p_{22}}\right\rangle=0$. From Eq. (37), the theoretical ratio to satisfy this constraint is $2.5: 2.5: 1$ for variants (2), (3) and (4), and the simulation result shown in Fig. 6b agrees this prediction. Finally, Table 4 lists the average strains $\left\langle\boldsymbol{\varepsilon}_{p_{11}}\right\rangle$ for different film orientations. The results show that this actuation strain is the largest for (110) films.

In the previous case, the orientation of the tunnel is along the $x_{2}$-direction, as illustrated in the left of Fig. $3 \mathrm{~b}$. Suppose now we change the design such that the tunnel's orientation is along the $x_{1}$-direction. In this case, the possible patterns of microstructure, under $\left\langle\sigma_{22}^{p}\right\rangle=\sigma^{0}=5 \mathrm{MPa}$, $\left\langle\varepsilon_{p_{12}}\right\rangle=0$ and $\left\langle\varepsilon_{p_{11}}\right\rangle=0$, are shown in Fig. 7a for (110) films and Fig. $7 \mathrm{~b}$ for (111) films. The explanations of the formation of these special patterns are similar to those in the previous case, and therefore are not given here. Note, though, that Fig. $7 \mathrm{~b}$ is different from Fig. 5b since the volume fractions of variants are different. The former has a ratio of 1:1:2 for variants (2), (3) and (4), while the latter shows equal volume fractions for these three variants. In this case, the tensile strain $\left\langle\boldsymbol{\varepsilon}_{p_{22}}\right\rangle$ is aimed to be maximized in order to achieve larger deformations. The results are listed in Table 4 for different film orientations. In contrast to the previous case, where the tunnel's orientation is per- pendicular to the present one, it is found that the actuation strain $\left\langle\boldsymbol{\varepsilon}_{p_{22}}\right\rangle$ is the largest for (111) films.

Finally, as depicted in the right of Figs. 5-7, it is interesting to see that all of the interfacial normals are almost compatible and follow the predictions listed in Tables 2 and 3 . Thus, the microstructure remains compatible during evolution under various loading conditions.

\section{Conclusions}

This article develops a novel multivariant model of the microstructure of martensite. The framework starts with the nonconventional choice of field variables motivated by the hierarchical structure of multirank laminates, as demonstrated in Fig. 2, to represent each variant. It offers an advantage of expressing the energy-well structure in a unified fashion, instead of choosing the special polynomial expansions of order parameters for a particular transformation. The resulting morphology is determined by balancing three competing forces: the first one, arising from the gradient energy, coarsens the microstructure; the second one, arising from the elastic strain energy, refines the microstructure; and the third one, arising from the anisotropy energy, selects the microstructure.

The framework is applied to the investigation of pattern formation in martensitic thin films. It chooses $\mathrm{Ti}-\mathrm{Ni}$ at the trigonal R-phase as the model material. Since the criterion for coherence is weakened in thin films and depends on the film normals, three common crystallographic orientations a

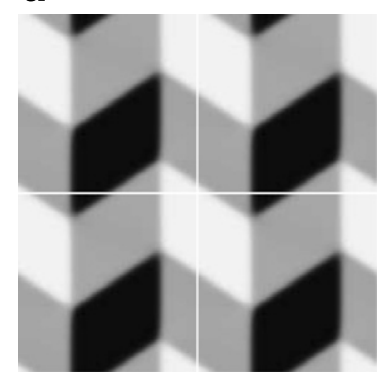

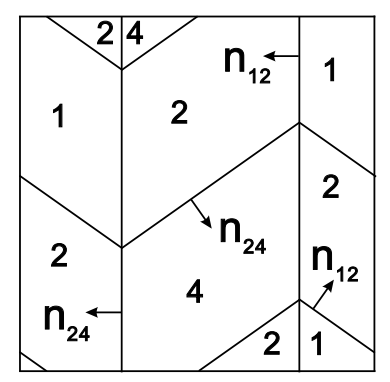

b

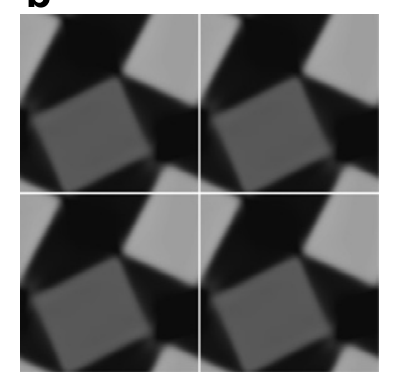

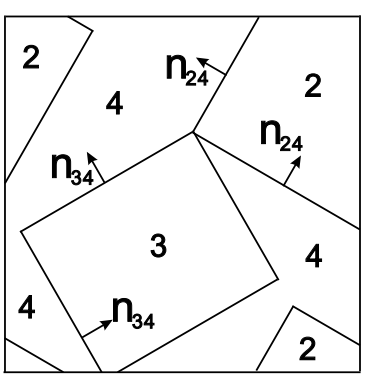

Fig. 7. Patterns of microstructure in another tunnel-shaped deformations: (a) for (1 1 10) films and (b) for (1 111$)$ films. The orientation of the tunnel is along the $x_{1}$-direction, in contrast to the one shown in Fig. $3 \mathrm{~b}$. All of the interfaces are compatibly oriented. 
are selected in simulations, including (001), (110) and (111) films. Fig. 4 highlights the striking contrast in selfaccommodation patterns for martensitic films with different orientations. Some of them have been observed in experiments. In addition, the interfaces separating different variants and volume fractions of each variant are found to be in excellent agreement with the theoretical predictions based on the Bhattacharya-James thin film theory [5].

As many thin film applications require the design of large strain microactuators, this motivates consideration of the optimal film microstructures and orientations to serve this goal. Fig. 5 lists various patterns for dome-shaped microactuators and concludes that (1 110$)$ films have the largest principal strains. On the other hand, the optimal film normals and patterns for tunnel-shaped microactuators depend on the orientation of the tunnel, as demonstrated in Figs. 6 and 7. It is also found that the resulting morphologies remain compatible under various loading conditions. This suggests that compatible walls provide a low-energy path for the evolution of microstructure, and understanding them leads to novel strategies of large strain actuation.

Finally, the simulation results demonstrate that the present framework is able to capture many important features for exploring the formation of martensitic patterns under various cases, and thus it is expected to be a valuable tool for designing advanced devices at the microscale.

\section{Acknowledgement}

The authors are pleased to acknowledge the financial support of the National Science Council of Taiwan under the Grant No. 96-2221-E-002-014.

\section{Appendix A. Elastic stress field in the Fourier space}

The solution of elastic stress due to periodic nonuniform transformation strain field can be obtained using the Fourier technique. In terms of the conventional Voigt notation, the stress vector $\tau=\left(\tau_{1}, \tau_{2}, \tau_{3}, \tau_{4}, \tau_{5}, \tau_{6}\right)$ denotes $\left(\boldsymbol{\sigma}_{11}, \boldsymbol{\sigma}_{22}\right.$, $\left.\boldsymbol{\sigma}_{33}, \boldsymbol{\sigma}_{23}, \boldsymbol{\sigma}_{31}, \boldsymbol{\sigma}_{12}\right)$, the strain vector $\boldsymbol{\epsilon}=\left(\epsilon_{1}, \epsilon_{2}, \epsilon_{3}, \epsilon_{4}, \epsilon_{5}, \epsilon_{6}\right)$ represents $\left(\varepsilon_{11}, \varepsilon_{22}, \varepsilon_{33}, 2 \varepsilon_{23}, 2 \varepsilon_{31}, 2 \varepsilon_{12}\right)$, and the transformation strain vector $\boldsymbol{\epsilon}^{*}=\left(\epsilon_{1}^{*}, \epsilon_{2}^{*}, \epsilon_{3}^{*}, \epsilon_{4}^{*}, \epsilon_{5}^{*}, \epsilon_{6}^{*}\right)$ represents $\left(\varepsilon_{11}^{*}, \varepsilon_{22}^{*}, \varepsilon_{33}^{*}, 2 \varepsilon_{23}^{*}, 2 \varepsilon_{31}^{*}, 2 \varepsilon_{12}^{*}\right)$. According to Eq. (10), they are related by $\tau=\mathbf{C}\left(\boldsymbol{\epsilon}-\epsilon^{*}\right)$, where $\mathbf{C}$ is the $6 \times 6$ stiffness matrix in terms of the Voigt notation. Notice that $\mathbf{C}^{\mathrm{T}}=\mathbf{C}$. In the two-dimensional case, $\tau$ becomes $\left(\tau_{1}, \tau_{2}, \tau_{6}\right), \epsilon$ and $\epsilon^{*}$ represent $\left(\epsilon_{1}, \epsilon_{2}, \epsilon_{6}\right)$ and $\left(\epsilon_{1}^{*}, \epsilon_{2}^{*}, \epsilon_{6}^{*}\right)$, and $\mathbf{C}$ here stands for the $3 \times 3$ stiffness matrix.

Let $\bar{f}(\xi)$ be the Fourier transform of any function $f(\mathbf{x})$, where $\mathbf{x}=\left(x_{1}, x_{2}, \ldots, x_{d}\right)$ and $\xi=\left(\xi_{1}, \xi_{2}, \ldots, \xi_{d}\right)$ are the coordinates in the real and reciprocal spaces, and $d$ is the space dimension. Both $f(\mathbf{x})$ and $\bar{f}(\xi)$ are related by

$\bar{f}(\boldsymbol{\xi})=\mathscr{F}[f]=\int_{-\infty}^{\infty} f(\mathbf{x}) \mathrm{e}^{-2 \pi \mathrm{i}(\xi \cdot \mathbf{x})} \mathrm{d} \mathbf{x}$

$f(\mathbf{x})=\mathscr{F}^{-1}[\bar{f}]=\int_{-\infty}^{\infty} \bar{f}(\xi) \mathrm{e}^{2 \pi \mathrm{i}(\xi \cdot \mathrm{x})} \mathrm{d} \xi$
Applying the Fourier transform on both Eq. (1) and Eq. (10) gives

$\overline{\mathbf{u}}=\left(\mathbf{B}^{\mathrm{T}} \mathbf{C B}\right)^{-1} \mathbf{B}^{\mathrm{T}} \mathbf{C} \overline{\boldsymbol{\epsilon}^{*}}$

where $\overline{\mathbf{u}}=\mathscr{F}[\mathbf{u}]$, and $\mathbf{B}$ is the matrix given by

$\mathbf{B}=2 \pi \mathrm{i}\left(\begin{array}{cc}\xi_{1} & 0 \\ 0 & \xi_{2} \\ \xi_{2} & \xi_{1}\end{array}\right) \quad$ if $d=2$

$\mathbf{B}=2 \pi i\left(\begin{array}{ccc}\xi_{1} & 0 & 0 \\ 0 & \xi_{2} & 0 \\ 0 & 0 & \xi_{3} \\ 0 & \xi_{3} & \xi_{2} \\ \xi_{3} & 0 & \xi_{1} \\ \xi_{2} & \xi_{1} & 0\end{array}\right) \quad$ if $d=3$

such that $\overline{\boldsymbol{\epsilon}}=\mathscr{F}[\boldsymbol{\epsilon}]=\mathbf{B} \overline{\mathbf{u}}$. As a result,

$$
\begin{aligned}
\bar{\tau} & =\mathscr{F}[\boldsymbol{\tau}]=\mathscr{F}\left[\mathbf{C}\left(\boldsymbol{\epsilon}-\boldsymbol{\epsilon}^{*}\right)\right]=\mathbf{C B} \overline{\mathbf{u}}-\mathbf{C} \overline{\boldsymbol{\epsilon}^{*}} \\
& =\left\{\mathbf{C B}\left(\mathbf{B}^{\mathrm{T}} \mathbf{C B}\right)^{-1} \mathbf{B}^{\mathrm{T}}-\mathbf{I}\right\} \mathbf{C} \overline{\boldsymbol{\epsilon}^{*}}
\end{aligned}
$$

where $\mathbf{I}$ is the identity matrix. The elastic stress field $\tau$ is therefore obtained by the inverse Fourier transform of $\bar{\tau}$ defined by Eq. (A2).

\section{Appendix B. Derivation of Eq. (20)}

Using Eq. (1) and the divergence theorem in Eq. (20) yields

$\int_{\Omega}\left(\boldsymbol{\sigma}-\boldsymbol{\sigma}^{0}\right) \cdot \delta \boldsymbol{\varepsilon}[\mathbf{u}] \mathrm{d} \mathbf{x}=\int_{\partial \Omega}\left(\boldsymbol{\sigma}-\boldsymbol{\sigma}^{0}\right) \mathbf{n} \cdot \delta \mathbf{u} \mathrm{d} S$

In deriving Eq. (B1), we have used the facts that $\nabla \cdot \boldsymbol{\sigma}=\mathbf{0}$, $\nabla \cdot \boldsymbol{\sigma}^{0}=\mathbf{0}$ and the elastic modulus $\mathbf{C}$ is symmetric. Now we show that Eq. (B1) vanishes according to two different cases: nonperiodic/periodic boundary conditions. First, under the nonperiodic setting, the definition of $\sigma^{0}$ requires it to be consistent with the traction boundary conditions, i.e. $\boldsymbol{\sigma} \mathbf{n}=\boldsymbol{\sigma}^{0} \mathbf{n}$ on the boundary where the applied traction is specified. On the other hand, $\delta \mathbf{u}=\mathbf{0}$ on the other part of the boundary where the displacement is specified. Combining these two observations gives $\int_{\partial \Omega}\left(\boldsymbol{\sigma}-\boldsymbol{\sigma}^{0}\right) \mathbf{n} \cdot \delta \mathbf{u d} S=0$ in Eq. (B1).

Second, under the periodic boundary condition, the domain $\Omega=\left(0, l_{0}\right)^{3}$ becomes a cube. If the overall strain is imposed, i.e. $\langle\boldsymbol{\varepsilon}\rangle=\boldsymbol{\varepsilon}^{0}$ is constant, then the term $\left(\boldsymbol{\sigma}^{0} \cdot \boldsymbol{\varepsilon}\right)$ is dropped from Eq. (13) and Eq. (B1) is changed to be

$\int_{\Omega} \boldsymbol{\sigma} \cdot \delta \boldsymbol{\varepsilon}[\mathbf{u}] \mathrm{d} \mathbf{x}=\int_{\Omega} \boldsymbol{\sigma} \cdot \delta \boldsymbol{\varepsilon}^{\prime}\left[\mathbf{u}^{\prime}\right] \mathrm{d} \mathbf{x}=\int_{\partial \Omega} \boldsymbol{\sigma} \mathbf{n} \cdot \delta \mathbf{u}^{\prime} \mathrm{d} S$

due to Eq. (14). $\mathbf{u}^{\prime}$ and $\sigma$ are periodic and the traction vector $\boldsymbol{\sigma n}$ evaluated at one side of the cube is in the opposite direction to that evaluated at an opposite face of the cube, giving rise to $\int_{\partial \Omega} \boldsymbol{\sigma n} \cdot \delta \mathbf{u}^{\prime} \mathrm{d} S=0$. However, if the boundary condition is imposed by assigning the overall stress, i.e. $\langle\boldsymbol{\sigma}\rangle=\boldsymbol{\sigma}^{0}$ is constant, more effort is needed to prove this 
argument. Indeed, using Eq. (14) and Eq. (17), Eq. (B1) can be rewritten as

$$
\begin{aligned}
\int_{\Omega} & \left(\boldsymbol{\sigma}-\boldsymbol{\sigma}^{0}\right) \cdot \delta \boldsymbol{\varepsilon}[\mathbf{u}] \mathrm{d} \mathbf{x} \\
= & \int_{\Omega} \boldsymbol{\sigma}^{\prime} \cdot \delta\langle\boldsymbol{\varepsilon}\rangle \mathrm{d} \mathbf{x}+\int_{\Omega} \boldsymbol{\sigma}^{\prime} \cdot \delta \boldsymbol{\varepsilon}^{\prime}\left[\mathbf{u}^{\prime}\right] \mathrm{d} \mathbf{x} \\
= & \delta\left(\int_{\Omega} \boldsymbol{\sigma}^{\prime} \cdot\langle\boldsymbol{\varepsilon}\rangle \mathrm{d} \mathbf{x}\right)-\int_{\Omega}\langle\boldsymbol{\varepsilon}\rangle \cdot \delta \boldsymbol{\sigma}^{\prime} \mathrm{d} \mathbf{x}+\int_{\partial \Omega} \boldsymbol{\sigma}^{\prime} \mathbf{n} \cdot \delta \mathbf{u}^{\prime} \mathrm{d} S \\
= & \delta\left(\langle\boldsymbol{\varepsilon}\rangle \cdot \int_{\Omega} \boldsymbol{\sigma}^{\prime} \mathrm{d} \mathbf{x}\right)-\langle\boldsymbol{\varepsilon}\rangle \cdot \delta\left(\int_{\Omega} \boldsymbol{\sigma}^{\prime} \mathrm{d} \mathbf{x}\right)+\int_{\partial \Omega} \boldsymbol{\sigma}^{\prime} \mathbf{n} \cdot \delta \mathbf{u}^{\prime} \mathrm{d} S
\end{aligned}
$$

The last term in Eq. (B3) is zero due to the similar reasons used to conclude the vanishing of Eq. (B2). The first two terms of Eq. (B3) are null since from Eq. (17), $\left\langle\boldsymbol{\sigma}^{\prime}\right\rangle=\mathbf{0}$. So is $\int_{\Omega} \boldsymbol{\sigma}^{\prime} \mathrm{d} \mathbf{x}=\mathbf{0}$.

\section{References}

[1] Bhattacharya K. Microstructure of martensite. Oxford: Oxford University Press; 2003.

[2] Wu SK, Lin HC. Mater Chem Phys 2000;64:81.

[3] Fu YQ, Du HJ, Huang WM, Zhang S, Hu M. Sens Actuator A Phys 2004;112:395.

[4] Bhattacharya K, James RD. Science 2005;307:53.

[5] Bhattacharya K, James RD. J Mech Phys Solids 1999;47:531.

[6] Shu YC. Arch Ration Mech Anal 2000;153:39.

[7] Bhattacharya K, DeSimone A, Hane KF, James RD, Palmstrøm CJ. Mater Sci Eng A 1999;273-275:685.

[8] Shu YC. Mater Trans 2002;43:1037.

[9] Wechsler MS, Lieberman DS, Read TA. J Metals 1953;197:1503.

[10] Bowles JS, MacKenzie JK. Acta Metall 1954;2:129.

[11] Ball JM, James RD. Arch Ration Mech Anal 1987;100:13.

[12] Khachaturyan AG. Theory of structural transformations in solids. New York: Wiley; 1983

[13] Roitburd AL. In: Ehrenreich H, Seitz F, Turnbull D, editors. Solid state physics, vol. 33. New York: Academic Press; 1978. p. 317.

[14] Kohn RV. Continuum Mech Thermodyn 1991;3:193.
[15] Bhattacharya K, Kohn RV. Arch Ration Mech Anal 1997;139:99.

[16] Shu YC, Bhattacharya K. Acta Mater 1998;46:5457.

[17] Wang Y, Khachaturyan AG. Acta Mater 1997;45:759.

[18] Artemev A, Jin Y, Khachaturyan AG. Acta Mater 2001;49:1165.

[19] Jin YM, Artemev A, Khachaturyan AG. Acta Mater 2001;49:2309.

[20] Slutsker J, Artemev A, Roytburd AL. J Appl Phys 2002;91:9049.

[21] Artemev A, Slutsker J, Roytburd AL. Acta Mater 2005;53:3425.

[22] Salje EKH. Phase transitions in ferroelastic and co-elastic crystals. Cambridge: Cambridge University Press; 1990.

[23] Lookman T, Shenoy SR, Rasmussen KØ, Saxena A, Bishop AR. Phys Rev B 2003;67:024114.

[24] Ahluwalia R, Lookman T, Saxena A, Albers RC. Acta Mater 2004;52:209.

[25] Chen LQ. Ann Rev Mater Res 2002;32:113.

[26] Wang J, Shi SQ, Chen LQ, Li Y, Zhang TY. Acta Mater 2004;52:749.

[27] Gao YF, Suo Z. J Appl Mech - Trans ASME 2002;69:419.

[28] Seol DJ, Hu SY, Li YL, Chen LQ, Oh KH. Mater Sci Forum 2002;408-412:1645.

[29] Jacobs AE, Curnoe SH, Desai RC. Phys Rev B 2003;68:224104.

[30] Levitas VI, Idesman AV, Preston DL. Phys Rev Lett 2004;93:105701.

[31] Dayal K, Bhattacharya K. Acta Mater 2007;55:1907.

[32] Shu YC, Yen JH. Appl Phys Lett 2007;91:021908.

[33] Bhattacharya K. Continuum Mech Thermodyn 1993;5:205.

[34] Shu YC, Yen JH, Chen HZ, Li JY, Li LJ. Appl Phys Lett 2008;92:052909.

[35] Li LJ, Li JY, Shu YC, Chen HZ, Yen JH. Appl Phys Lett 2008;92:172504.

[36] Shu YC, Yen JH, Shieh J, Yeh JH. Appl Phys Lett 2007;90: 172902.

[37] Yen JH, Shu YC, Shieh J, Yeh JH. J Mech Phys Solids 2008;56:2117.

[38] Lu W, Suo Z. J Mech Phys Solids 2001;49:1937.

[39] Li JY, Luo Y, Bai M, Ducharme S. J Mech Phys Solids 2006;54:2162.

[40] Miyazaki S, Kimura S, Otsuka K. Philos Mag A 1988;57:467.

[41] Tomozawa M, Kim HY, Miyazaki S. J Intell Mater Syst Struct 2006;17:1049.

[42] Shu YC, Lin MP, Wu KC. Mech Mater 2004;36:975.

[43] Shu YC. J Elast 2002;66:63.

[44] Chen LQ, Shen J. Comput Phys Commun 1998;108:147.

[45] Otsuka K, Wayman CM. Shape memory materials. Cambridge: Cambridge University Press; 1998.

[46] Fukuda T, Saburi T, Doi K, Nenno S. Mater Trans JIM 1992;33:271.

[47] Streiffer SK, Parker CB, Romanov AE, Lefevre MJ, Zhao L, Speck JS, et al. J Appl Phys 1998;83:2742. 